\title{
HI distribution and kinematics of NGC 1569
}

\author{
J. M. Stil ${ }^{1,2}$ and F. P. Israel ${ }^{1}$ \\ 1 Leiden Observatory, PO Box 9513, 2300 RA Leiden, The Netherlands \\ 2 Department of Physics and Astronomy, University of Calgary, Calgary, Alberta, Canada
}

Received 26 March 2002 / Accepted 20 June 2002

\begin{abstract}
We present WSRT observations of high sensitivity and resolution of the neutral hydrogen in the starburst dwarf galaxy NGC 1569. Assuming a distance of $2.2 \mathrm{Mpc}$, we find a total HI mass of $1.3 \times 10^{8} M_{\odot}$ to be distributed in the form of a dense, clumpy ridge surrounded by more extended diffuse HI containing a few additional discrete features, such as a Western HI Arm and an HI bridge reaching out to a small counterrotating companion cloud. About 10\% by mass of all HI in NGC 1569 is at unusually high velocities. Some of this HI may be associated with the mass outflow evident from $\mathrm{H} \alpha$ measurements, but some may also be associated with NGC 1569's HI companion and intervening HI bridge, in which case, infall rather than outflow might be the cause of the discrepant velocities. No indication of a large bubble structure was found in position-velocity maps of the high-velocity HI. The galaxy as a whole is in modest overall rotation, but the HI gas lacks any sign of rotation within $60^{\prime \prime}(0.6 \mathrm{kpc})$ from the center, i.e. over most of the optical galaxy. Here, turbulent motions resulting from the starburst appear to dominate over rotation. In the outer disk, the rotational velocities reach a maximum of $35 \pm 6 \mathrm{~km} \mathrm{~s}^{-1}$, but turbulent motion remains significant. Thus, starburst effects are still noticeable in the outer HI disk, although they are no longer dominant beyond $0.6 \mathrm{kpc}$. Even excluding the most extreme high-velocity HI clouds, NGC 1569 still has an unusually high mean HI velocity dispersion of $\sigma_{\mathrm{v}}=21.3 \mathrm{~km} \mathrm{~s}^{-1}$, more than double that of other dwarf galaxies.
\end{abstract}

Key words. ISM: atoms - galaxies: individual: NGC 1569 - irregular - kinematics and dynamics - starburst

\section{Introduction}

NGC 1569 is a small Im type galaxy at a distance of $2.2 \pm$ $0.6 \mathrm{Mpc}$ (Israel 1988), and a probable member of the IC 342 group (Huchtmeier et al. 2000). With a maximum optical size of $2.9^{\prime}(1.85 \mathrm{kpc})$, NGC 1569 is dominated by the aftermath of a burst of star formation. Assuming a Salpeter IMF with slope 2.35 and lower and upper mass cut-offs at 0.1 and $120 M_{\odot}$ respectively, Greggio et al. (1998) found a star formation rate of $0.5 M_{\odot} \mathrm{yr}^{-1}$ with little change over the past 150 Myr. Taking into account the limited size and mass of NGC 1569 , this is a very high rate (Israel 1980). Although the present star formation rate is still high, the most intense starburst phase occurred between 5 and 10 Myr ago (Israel \& de Bruyn 1988; Vallenari \& Bomans 1996; Greggio et al. 1998). The high star formation rates imply a type II supernova production corresponding to a total of $2-3 \times 10^{5}$ over the past $100 \mathrm{Myr}$ in this small volume of space.

Outflow of gas from NGC 1569 is inferred from the kinematics of an extended system of $\mathrm{H} \alpha$ filaments (De Vaucouleurs et al. 1974; Waller 1991; Heckman et al. 1995; Martin 1998) and from extended X-ray emission (Heckman et al. 1995). NGC 1569 is surrounded by an extended halo of relativistic electrons emitting synchrotron radiation (Israel \& De Bruyn 1988).

Send offprint requests to: J. M. Stil, e-mail: stil@ras . ucalgary.ca
NGC 1569 contains three very luminous, compact star clusters designated NGC 1569 A, B and C. NGC 1569 A may be a double cluster; the mass of its brightest component is about $3 \times 10^{5} M_{\odot}$ (Ho \& Filippenko 1996; De Marchi et al. 1997). Given its mass, luminosity and colour, NGC 1569 A will closely resemble a globular cluster in the Milky Way after an elapsed time of $15 \mathrm{Gyr}$.

In a previous paper, we have presented evidence for interaction with a massive nearby HI cloud, NGC 1569 -HI. This cloud is probably related to the recent starburst in NGC 1569 , whereas the nearby dwarf companion UGCA 92 almost certainly is not (Stil \& Israel 1998).

Observations of NGC 1569 in the $21-\mathrm{cm}$ line of atomic hydrogen (HI) have been presented by Reakes (1980) and by Israel \& Van Driel (1990). Their maps show the HI structure to consist mainly of a high column-density ridge with three local maxima and an arm-like feature extending approximately $3^{\prime}$ to the west. The maximum rotational velocity of NGC 1569 is about $30 \mathrm{~km} \mathrm{~s}^{-1}$. Israel \& Van Driel (1990) also found a region with a local velocity dispersion comparable to the rotational velocity.

\section{Observations and data reduction}

We observed the HI distribution in NGC 1569 with the WSRT during four 12-hour runs between November 1989 and January 1990. We sampled a total of 160 baselines ranging from $36 \mathrm{~m}$ 
to $2772 \mathrm{~m}$ with increments of $18 \mathrm{~m}$. The $2.5 \mathrm{MHz}$ passband was sampled by 128 independent frequency channels of $4.1 \mathrm{~km} \mathrm{~s}^{-1}$ width, yielding a Hanning-smoothed velocity resolution of $8.2 \mathrm{~km} \mathrm{~s}^{-1}$. Standard gain and phase corrections were applied by the reduction group in Dwingeloo. First inspection of the data, flagging of bad data and fourier transformation was done in the NEWSTAR package. We produced maps at resolutions of $13.5^{\prime \prime}, 27^{\prime \prime}$ and $60^{\prime \prime}$ by applying Gaussian weight functions with respective widths in the UV-plane of $1960 \mathrm{~m}$, $843 \mathrm{~m}$ and $390 \mathrm{~m}$. Resulting dirty maps were imported into the GIPSY package for further reduction and analysis. At each pixel, we subtracted continuum emission by fitting a first-order polynomial to a total of 48 line-free channels on both sides of the frequency band. We then identified manually in each channel map those areas containing line emission. Within these areas, the CLEANing algorithm (Högbom 1974) searched for components and subtracted scaled antenna patterns from the map down to levels of 0.5 the rms noise in a single channel map. The rms noise per channel after Hanning-smoothing is $1.1 \mathrm{mJy}$ per beam at $27^{\prime \prime}$ resolution. We allowed both positive and negative CLEAN components in order to minimize a bias for positive components at low intensity levels. As interferometer spacings shortwards of $36 \mathrm{~m}$ are lacking, structures larger than a few arcminutes are increasingly diluted. As the $\mathrm{HI}$ emission in individual channel maps rarely extends that far, this effect can safely be ignored.

\section{Results and analysis}

\section{1. $21 \mathrm{~cm}$ continuum}

We show the distribution of radio continuum emission in NGC 1569 at resolutions of $13.5^{\prime \prime}$ and 60" in Fig. 1. In the high-resolution map, the two peaks coincide within 4 " with the most luminous HII regions labeled 2 and 3 (peak at $\alpha_{1950}=$ $\left.4^{\mathrm{h}} 26^{\mathrm{m}} 2^{\mathrm{s}} .7, \delta_{1950}=64^{\circ} 44^{\prime} 32^{\prime \prime}\right)$ and 7 (peak at $\alpha_{1950}=4^{\mathrm{h}} 26^{\mathrm{m}} 7^{\mathrm{s}} .0$, $\delta_{1950}=64^{\circ} 44^{\prime} 20^{\prime \prime}$ ) by Waller (1991). The continuum map also shows a curved extension at the position of the H $\alpha$ arm. The extended radio continuum halo has become visible in the $60^{\prime \prime}$ resolution map. The integrated $21-\mathrm{cm}$ continuum flux-density of NGC 1569 measured in the $60^{\prime \prime}$ resolution map is $438 \pm 5 \mathrm{mJy}$, consistent with the results summarized in Israel \& De Bruyn (1988).

\section{2. $21 \mathrm{~cm}$ line profile and $\mathrm{HI}$ mass}

We obtained the spatially-integrated HI line profile of NGC 1569 (Fig. 2) by summing the flux in each channelmap. In order to minimize the effect of sidelobes of the unCLEANed Galactic foreground emission, we used in the summation only those regions that were also selected for CLEANing. The asymmetry in the line profile is not due to absorption by $\mathrm{HI}$ in the Galactic foreground, as this would require significant optical depths in the velocity range $-70 \mathrm{~km} \mathrm{~s}^{-1}$ to $-30 \mathrm{~km} \mathrm{~s}^{-1}$, which are not observed (see Stil \& Israel 1998, their Fig. 4). The HI line flux between $v_{\text {hel }}=-190 \mathrm{~km} \mathrm{~s}^{-1}$ and $v_{\text {hel }}=$ $-10 \mathrm{~km} \mathrm{~s}^{-1}$, corrected for primary beam attenuation and including NGC 1569-HI (Stil \& Israel 1998) is $116 \mathrm{Jy} \mathrm{km} \mathrm{s}^{-1}$.
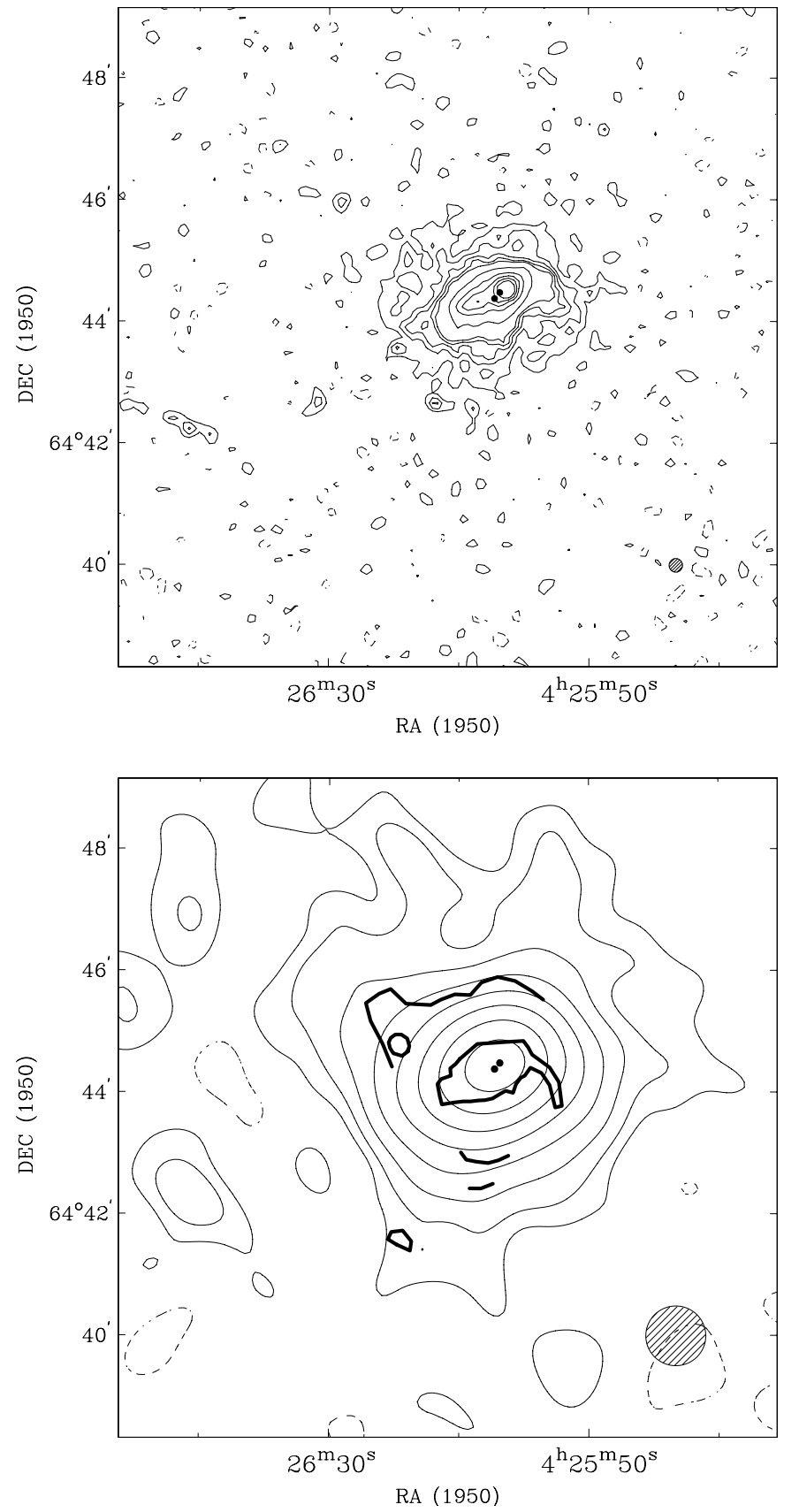

Fig. 1. Continuum maps of NGC 1569. Top: resolution 13" and contour levels at -0.5 (dashed), $0.5(2 \sigma), 1.0,1.5,2.0,2.5,3.0,3.5,4.0$, $4.5,5.0,10.0,15.0,20.0,25.0 \mathrm{mJy} / \mathrm{beam}$. The two dots near the center mark the respective locations of super star clusters A and B. Bottom: resolution $60^{\prime \prime}$ and contour levels at $-1.0,1.0(2 \sigma), 2.0,4.0,8.0,16.0$, $32.0,64.0,128.0 \mathrm{mJy} / \mathrm{beam}$. Clusters A and B are marked also in this map as well as the outlines of $\mathrm{H} \alpha$ emission. In both maps, synthesized FWHM beamsizes are indicated by hatched circles.

At the distance of $2.2 \mathrm{Mpc}$, the HI mass of NGC 1569 is thus $M_{\mathrm{HI}}=1.3 \times 10^{8} M_{\odot}$, in good agreement with the values listed by Israel (1988).

The intensity-weighted mean velocity of the line profile is $v_{\text {sys }}=-90.3 \mathrm{~km} \mathrm{~s}^{-1}$, i.e identical to the value $v_{\text {sys }}=-89 \mathrm{~km} \mathrm{~s}^{-1}$ listed in the RC3 (de Vaucouleurs et al. 1991), but approximately $13 \mathrm{~km} \mathrm{~s}^{-1}$ smaller than the systemic velocity measured 


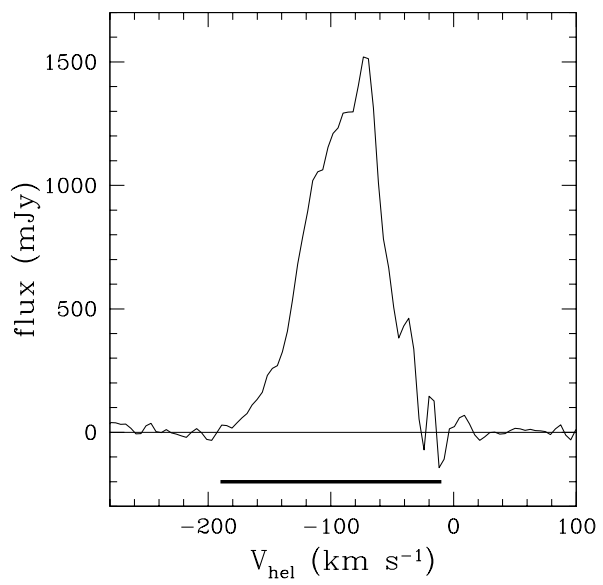

Fig. 2. Spatially integrated HI emission line profile of NGC 1569. The horizontal line indicates the velocity range over which the line flux was integrated.

either in $\mathrm{H} \alpha$ (Tomita et al. 1994) or from the HI velocity field (cf. Reakes 1980). This discrepancy probably reflects the asymmetry of the HI line profile (see also Sect. 3.5). The presence of small-scale emission from the Galactic foreground produces a negative offset of maximum $-0.7 \mathrm{mJy} /$ beam $(0.5 \sigma)$ in the two most affected channels at $-57 \mathrm{~km} \mathrm{~s}^{-1}$ and $-16 \mathrm{~km} \mathrm{~s}^{-1}$. We have not attempted to reduce the influence of Galactic foreground emission by eliminating the shortest baselines, as this would further dilute any large-scale structures in NGC 1569.

\subsection{Overall HI distribution}

Figure 3 shows the distribution of HI column densities at $13^{\prime \prime}$ and $27^{\prime \prime}$ resolution. The low-resolution map shows both NGC 1569 and NGC 1569-HI (Stil \& Israel 1998). The highresolution map shows only the central region with the high column-density ridge. In constructing these maps, we applied the areas selected for the CLEAN algorithm as a mask to the data and then integrated the flux over the observed velocity range. In this way, we both suppress noise contributions from empty channels and avoid the bias against low column-density components that would be introduced by discarding pixels below some intensity threshold.

The overall HI morphology is S-shaped with NGC 1569-HI and the HI Bridge extending to the southeast. At the other side, a feature which we will call the Western HI Arm borders a region of low HI column-densities extending to the north-west. The Western HI Arm should not be confused with the $\mathrm{H} \alpha$ arm, which is much smaller and nearer to the starclusters A and B. It is not a smooth feature, but consists of a chain of four to six individual components. The high-resolution map also reveals the clumpy nature of the main HI ridge. However, significant amounts of molecular hydrogen probably fill most of the gap between the two brightest HI maxima (Lisenfeld et al. 2002). The optical galaxy is closely associated with this main ridge. Figure 3 shows that the HI hole found by Israel \& Van Driel (1990) surrounding super starclusters A and B (marked by dots) connects with a distinct HI depression centered on $\alpha=$ $4^{\mathrm{h}} 26^{\mathrm{m}} 6^{\mathrm{s}}, \delta=64^{\circ} 44^{\prime}$. This is bordered in the south by an
HI structure coincident with the $\mathrm{H} \alpha$ arcs described by Waller (1991). Here, the HI bridge apparent in the low-resolution map is projected onto the main body of NGC 1569. Another, smaller HI minimum at $\alpha_{1950}=4^{\mathrm{h}} 26^{\mathrm{m}} 15^{\mathrm{s}} .6, \delta_{1950}=64^{\circ} 44^{\prime} 00^{\prime \prime}\left( \pm 5^{\prime \prime}\right.$ in either direction) appears to be an actual hole in the HI distribution. At its southwestern edge, a shell-like HII region centered on a Wolf-Rayet star occurs (Drissen \& Roy 1994).

The large-scale HI morphology and kinematics are reminiscent of tidal distortion of the outer regions of NGC 1569. However, a major problem with this interpretation is that this requires a relatively massive, nearby companion that has not been detected.

\subsection{Velocity field and velocity gradient}

In Fig. 4, we show the map of intensity-weighted mean velocities. As before, we used the areas selected for the CLEAN algorithm to suppress noise from regions without detectable line emission. The overall velocity field clearly shows the rotation of NGC 1569 and the apparent retrograde velocity of NGC 1569-HI (cf. Stil \& Israel 1998). However, on smaller scales, the velocity field exhibits significant local departures from an axially symmetric rotating disk. Most of the smallscale structure in the velocity field can be traced back to peculiar structures visible in individual channel maps. For example, the strongest irregularity is a steep velocity gradient near $\alpha_{1950}=4^{\mathrm{h}} 25^{\mathrm{m}} 51^{\mathrm{s}} .4, \delta_{1950}=64^{\circ} 44^{\prime} 15^{\prime \prime}$ which resulted from the superposition of $\mathrm{HI}$ in regular rotation and an $\mathrm{HI}$ cloud with a peculiar velocity readily identified in the channel maps and already noted by Israel \& Van Driel (1990) for its high HI velocity. A small east-west velocity gradient is observed in NGC 1569-HI but it is too chaotic to provide a convincing case for rotation. Along the bridge, the velocity changes gradually to the velocity of NGC 1569 (Stil \& Israel 1998).

In Fig. 5 we show position-velocity maps along lines parallel to the major axis of NGC 1569. Although starcluster A is not necessarily the dynamic center of NGC 1569 , it provides a useful reference point and for this reason was chosen for the zeropoint of the spatial axis in the central panel. The rotation of NGC 1569 is clearly visible as the velocity gradient defined by the locus of bright emission in the central panel. Note that the region of high velocity-dispersion near starcluster $\mathrm{A}$, referred to above, is most prominent in the central panel. We will return to this feature below.

The kinematic signature of the HI bridge is apparent in the $\mathrm{p}$-V diagrams at velocities around $100 \mathrm{~km} \mathrm{~s}^{-1}$ and at offsets of $2^{\prime \prime}$ or more along the major axis. The p-V diagrams $90^{\prime \prime}$ and $120^{\prime \prime}$ south of starcluster A intersect the region of the $\mathrm{H} \alpha$ arcs. In these panels, $\mathrm{HI}$ at $1^{\prime \prime}$ east of starcluster A has a velocity gradient opposed to the overall rotation of NGC 1569. This inverted gradient occurs in a small region where the emission of the HI Bridge blends into that of NGC 1569. This is also the location where the HI bridge might be physically connected to NGC 1569, if such a physical connection in fact exists. 

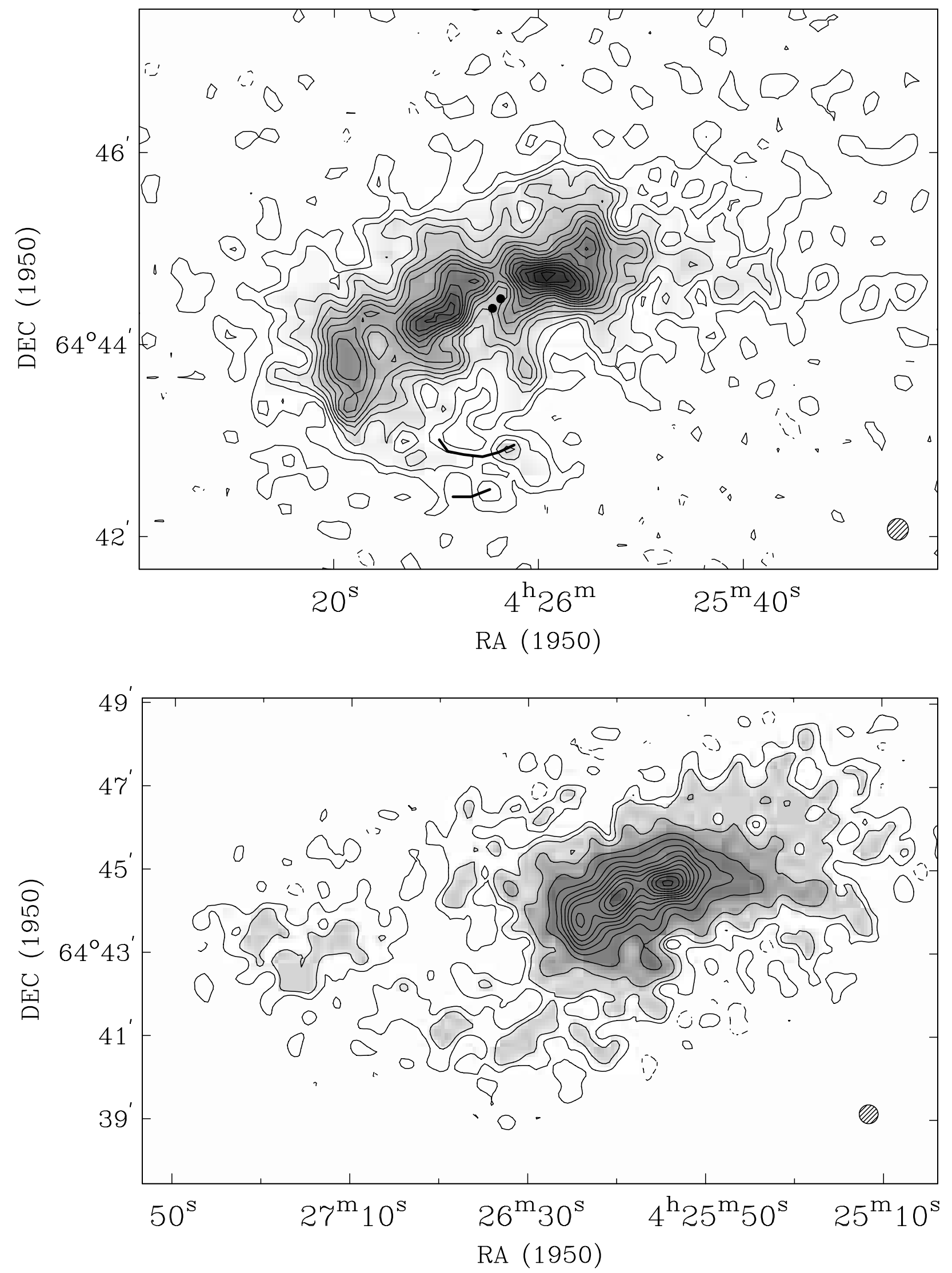

Fig. 3. HI column density maps of NGC 1569 . Top: map at $13^{\prime \prime}$ resolution with contours at -5 (dashed), $5,10,15,20, \ldots \times 10^{20} \mathrm{HI} \mathrm{cm}^{-2}$. Linear grayscales start at $10.0 \times 10^{20} \mathrm{HI} \mathrm{cm}^{-2}$. The two dots near the center mark the position of starclusters A and B. The curved lines mark the position of Arc 1 and Arc 2 (Waller 1991). Bottom: map at 27" resolution with contours at -1 (dashed), 1, 2, 4, 9, 14, 19, $24 \ldots \times 10^{20} \mathrm{HI} \mathrm{cm}^{-2}$. Grayscales change at 2.0, 4.0, 8.0, 50.0, and $70.0 \times 10^{20} \mathrm{HI} \mathrm{cm}^{-2}$. 


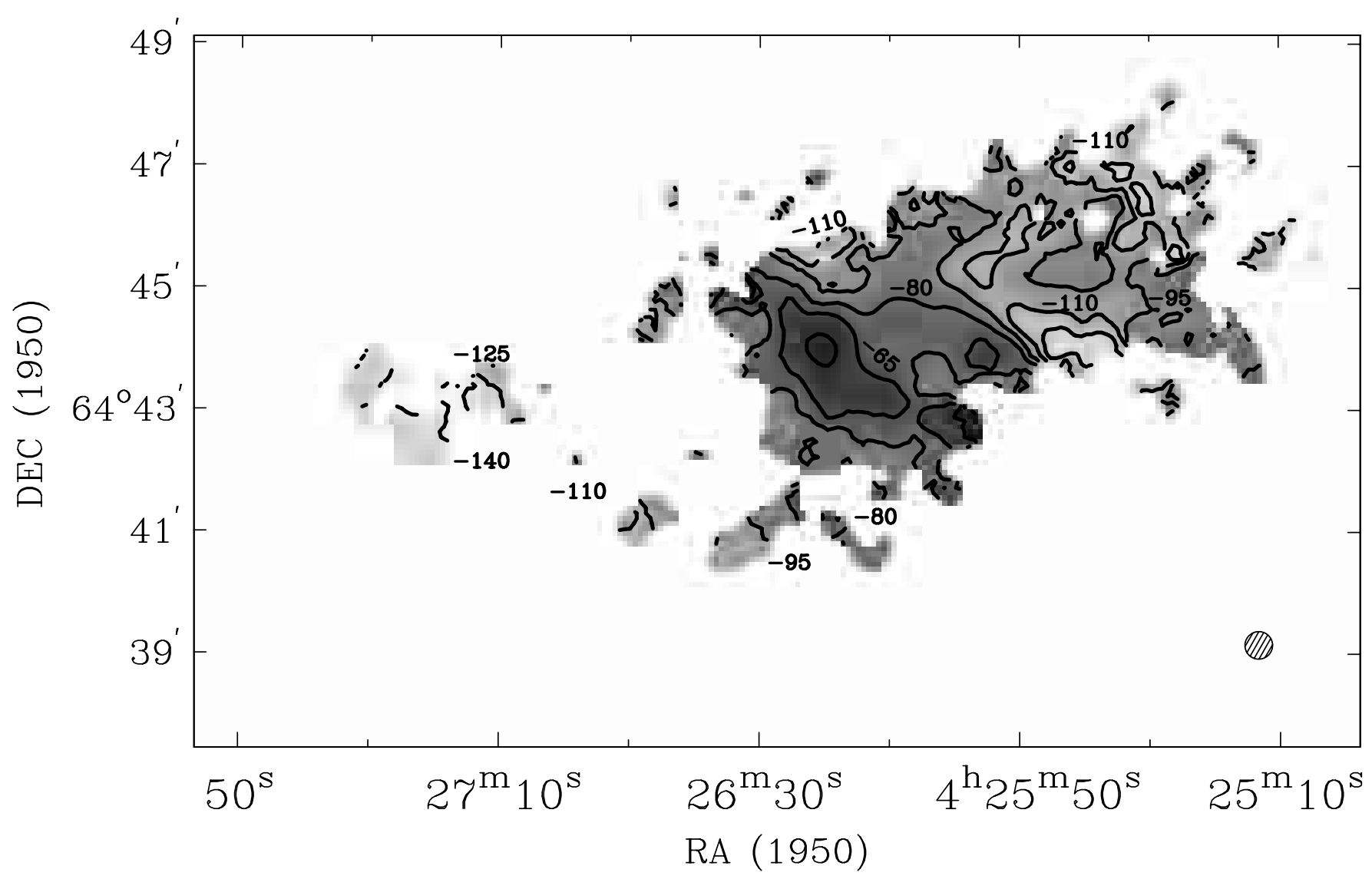

Fig. 4. Velocity field of NGC 1569 at $27^{\prime \prime}$ resolution. Lines of constant velocity, included at intervals of $15 \mathrm{~km} \mathrm{~s}^{-1}$, are marked by their heliocentric velocity.

\subsection{Rotation curve}

Because of the large departures from circular motion, the velocity field of NGC 1569 cannot be analyzed with methods presupposing regular rotation, such as the method of tilted-ring fitting. Before we can accurately determine the rotation curve, we must first exclude the high velocity gas from the analysis. By fitting ellipses to the regularly shaped outer isophotes of an $R$-band image of NGC 1569 taken with the 1-metre Jacobus Kapteyn Telescope at the Roque de Los Muchachos Observatory, we determined the optical center and axial ratio of NGC 1569 and used them to define the dynamic center and the inclination required for the analysis. The mean center of isophotes with semi-major axis between $r=50^{\prime \prime}$ and $r=75^{\prime \prime}$ is $\alpha_{1950}=4^{\mathrm{h}} 26^{\mathrm{m}} 4.5, \delta_{1950}^{\mathrm{s}}=64^{\circ} 44^{\prime} 26^{\prime \prime}$ (rms scatter of $2^{\prime \prime}$ in either coordinate). The astrometry was tied to the positions of the starclusters A and B given by Waller (1991). The position angle of the major axis is $117^{\circ} \pm 2^{\circ}$ (counterclockwise) with an axial ratio $q=0.53 \pm 0.04$. Assuming symmetry around the minor axis and an intrinsic thickness $q_{0}=0.3$, the observed axial ratio translates into an inclination $i=63^{\circ}$. The shape of the HI distribution, although more poorly defined, is consistent with these parameters (see also Reakes 1980), in particular as the directions of the HI major axis, the optical major axis and the overall velocity gradient coincide.
To determine the rotation curve of NGC 1569, we followed a procedure derived from the classic tilted-ring analysis. We divided the galaxy into concentric annuli with the center and axial ratio derived above. Along these annuli we then constructed p-V maps with the GIPSY task ELLPROF (Fig. 6), sampling radii ranging from $15^{\prime \prime}$ to $180^{\prime \prime}$ at $15^{\prime \prime}$ intervals. For radii $\leq 60^{\prime \prime}$ we used the high-resolution $13^{\prime \prime} .5$ in order to reduce the adverse effects of beamsmearing, while for larger radii we used the $27^{\prime \prime}$ data in order to increase the signal-to-noise ratio.

For a single annulus and circular orbits, the observed velocity as a function of position angle is

$v_{\mathrm{obs}}(\cos \theta)=v_{\mathrm{sys}}+v(r) \sin i \cos \theta$

with $v_{\text {sys }}$ the systemic velocity, $v(r)$ the rotation velocity at radius $r$, and the position angle $\theta$ measured in the plane of the ring, from the major axis at the receding side of the galaxy.

The general $\cos \theta$ arrangement of the emission in Fig. 6 confirms the large scale rotation with localized but very significant departures from rotation. Peculiar velocities similar in magnitude to the rotation velocity itself, are clearly visible in Fig. 6. A good example is the region of high velocity dispersion already mentioned, at $r \approx 90^{\prime \prime}, \theta=140^{\circ}$. Likewise, the peculiar velocity of the Western Arm is clearly visible at radii $r \geq 90^{\prime \prime}$ between $\theta=170^{\circ}$ and $\theta=220^{\circ}$. These and similar features are further discussed in Sect. 3.7. 


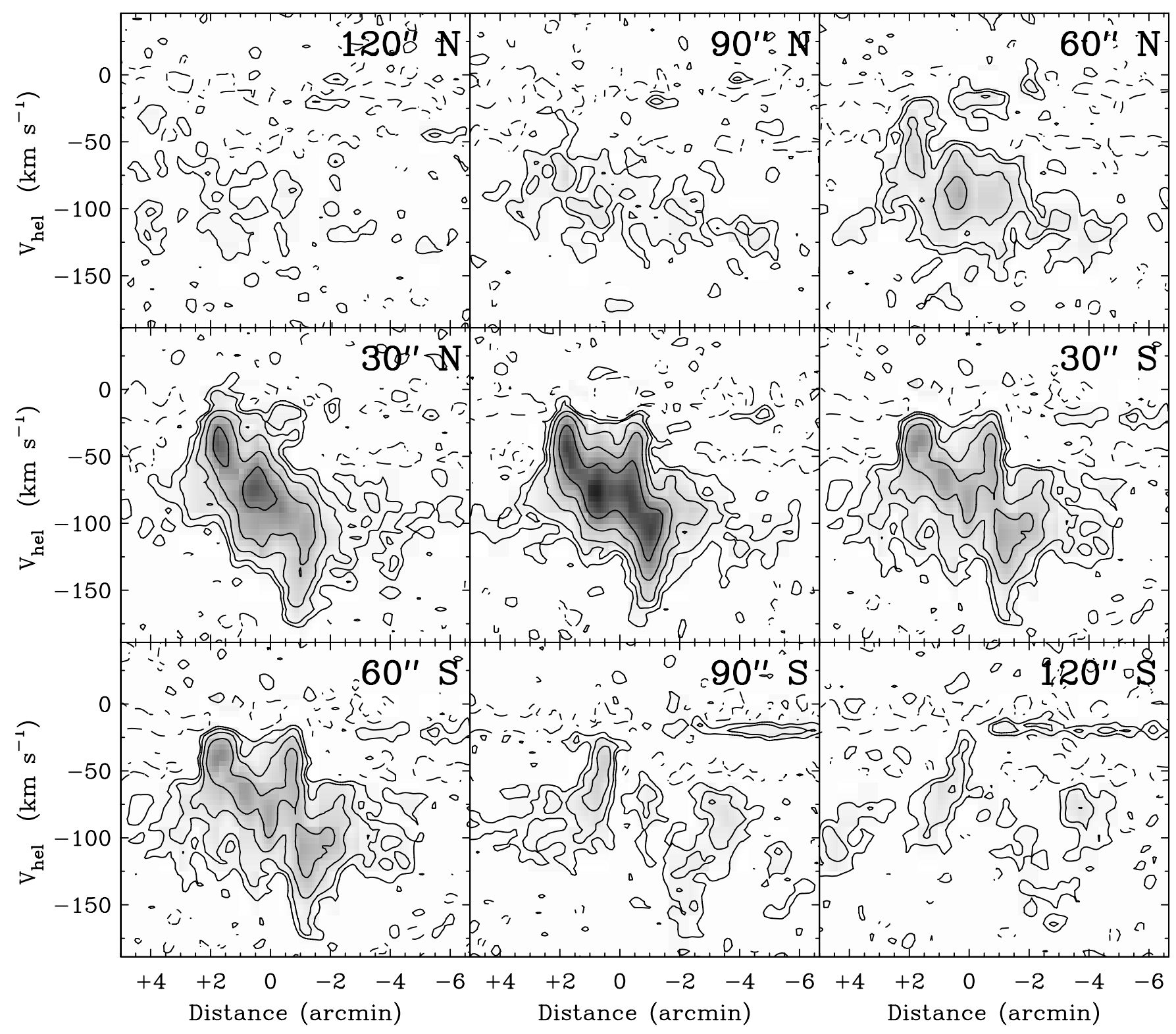

Fig. 5. Position-velocity maps along lines parallel to the major axis of NGC 1569 (position angle $116^{\circ}$ ). The central position of the central panel coincides with starcluster A. In all other panels, offsets from the major axis are given in the upper right corner. Contours are at $-2.2 \mathrm{mJy}$ (dashed), $2.2 \times 2^{n}(n=0,1,2, \ldots) \mathrm{mJy}$, linear grayscales start at $2.2 \mathrm{mJy}$.

The velocity $v_{\text {obs }}(\cos \theta)$ was measured by fitting a single Gaussian profile to the data at intervals corresponding to approximately half the FWHM beamsize. For larger annuli, more independent points are available (see the first four panels in Fig. 6). Areas with clear departures from the general $\cos (\theta)$ behaviour were avoided. A least-squares fit of $v_{\text {obs }}$ as a function of $\cos (\theta)$ yielded values of $v_{\text {sys }}$ and $v(r) \sin i$ for each annulus.

The fits of Eq. (1) to the data are shown in Fig. 7. The formal errors in the velocities are a few $\mathrm{km} \mathrm{s}^{-1}$. However, the errorbars in Fig. 7 were defined as half the fitted velocity dispersion. This is a conservative estimate of the error in the central velocity introduced by the blending of two equalamplitude Gaussian components which cannot be separated by visual inspection. For radii more than $150^{\prime \prime}$, the signal-to-noise ratio and the importance of anomalous velocity components (the Western HI Arm and the bridge) did not allow an unambiguous determination of the rotation velocity. However, a tentative rotation velocity at $r=180^{\prime \prime}$ was derived from the $60^{\prime \prime}$ resolution data. Table 1 lists the systemic velocity and the rotation velocity for the fits shown in Fig. 7. The mean systemic velocity of all radii is $-81.6 \pm 4.4 \mathrm{~km} \mathrm{~s}^{-1}$, where the error is the rms scatter around the mean. This is consistent with $v_{\text {sys }}=77 \pm 1 \mathrm{~km} \mathrm{~s}^{-1}$ found by Reakes (1980) from the HI velocity field and by Tomita et al. (1994) from $\mathrm{H} \alpha$ kinematics, but $9 \mathrm{~km} \mathrm{~s}^{-1}$ smaller than the systemic velocity derived from the $21 \mathrm{~cm}$ line profile. Separate fits to the receding and approaching side of NGC 1569 are also listed. The errors in Table 1 represent the rms fit residuals. However, half the velocity 

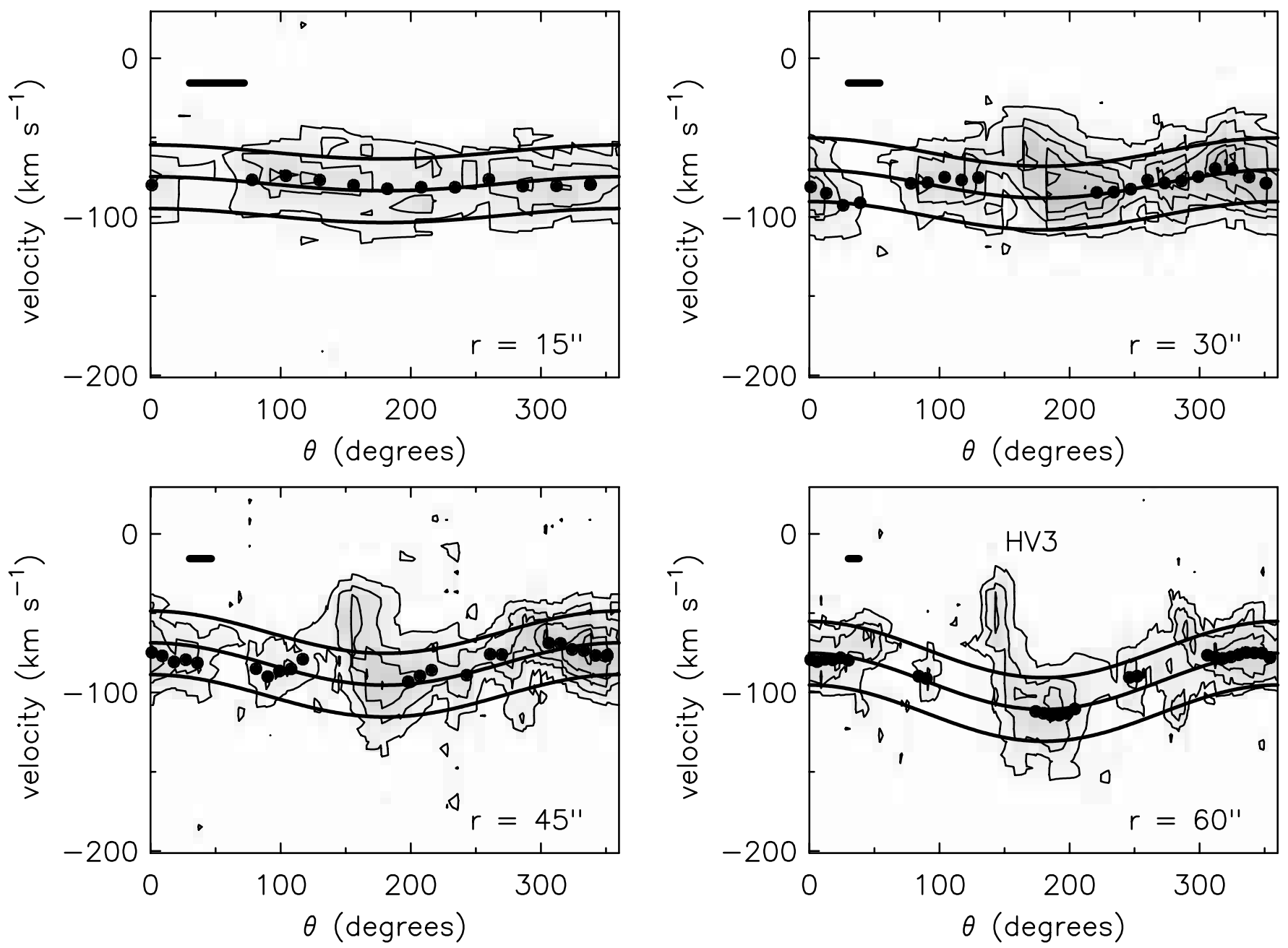

Fig. 6. Position-velocity maps along concentric ellipses defined by the optical isophotes. The angle $\theta=0^{\circ}$ corresponds to the major axis at the receding side (east) of NGC 1569. The first four panels $\left(r \leq 60^{\prime \prime}\right)$ were constructed from the high-resolution data cube (13" FWHM beam), the remainder from the smoothed data (27" FWHM beam). Contours are at positive multiples of $5 \sigma$, where $\sigma=0.97 \mathrm{mJy} /$ beam (high resolution) and $\sigma=1.1 \mathrm{mJy} /$ beam (low resolution) is the rms noise in a channel map. Grayscales start at $1.65 \mathrm{mJy} / \mathrm{beam}$. A horizontal bar in the first four panels indicates the angle occupied by the 13". 5 (FWHM) beam as seen from the center. Dots mark the velocity of Gaussians fitted to the line profile. The nomenclature of gas with anomalous velocities is defined in Fig. 12 and Table 2. Foreground Galactic emission is labeled "G". The solid curves indicate rotation velocities as explained in the text. Figure continued on following pages.

difference between the approaching and receding side is probably a more realistic estimate of the uncertainty of the rotation velocity. The rotation curve thus derived is shown graphically in Fig. 8. The difference between the rotation curves of the approaching and the receding side is mainly due to limited sampling imposed by the necessity to avoid contamination by high-velocity HI.

Rotation velocitiies for $r>60^{\prime \prime}$ are in good agreement with the rotation curve of Reakes (1980), which rises linearly with radius to a maximum of $25 \mathrm{~km} \mathrm{~s}^{-1}$ (not corrected for inclination) at $2^{\prime}$ from the center, where it appears to turn over. The subsequent decrease is, however, only marginally significant. We confirm the suspicion of Reakes (1980) that the decline in his rotation curve is due to HI with anomalous velocities.

If we were to assume a linearly rising rotation curve out to radii of $r=75^{\prime \prime}$, the inferred rotational velocities are 4.5, 9, and $13.5 \mathrm{~km} \mathrm{~s}^{-1}$ at $r=15^{\prime \prime}, 30^{\prime \prime}$ and $45^{\prime \prime}$ (see the cosine curves in the first four panels of Fig. 6 and the dashed line in Fig. 7. The actual data at these radii, however, indicate rotational velocities that are systematically smaller. In fact, all rotational velocities for $r \leq 60^{\prime \prime}$ are consistent with zero (Figs. 6 and 7). A lack of rotation in the same region is also evident from Fig. 5. Beamsmearing is an unlikely cause because such a discrepancy is not normally found in dwarf galaxies observed at similar resolutions (Stil \& Israel 2002). Moreover, a lack of rotation in this region was also deduced by Castles et al. (1991) and Tomita et al. (1994) from $\mathrm{H} \alpha$ where beamsmearing plays no role. Figure 6 shows that very low rotational velocities are representative for all inner $\mathrm{HI}$, and not caused by local highvelocity components either. The data are consistent with little or no rotation for all values of $\theta$.

The mean HI velocity dispersion derived from the Gaussian fits, excluding the regions with high-velocity gas, is $21.3 \mathrm{~km} \mathrm{~s}^{-1}$. This is unusually high. In a sample of 

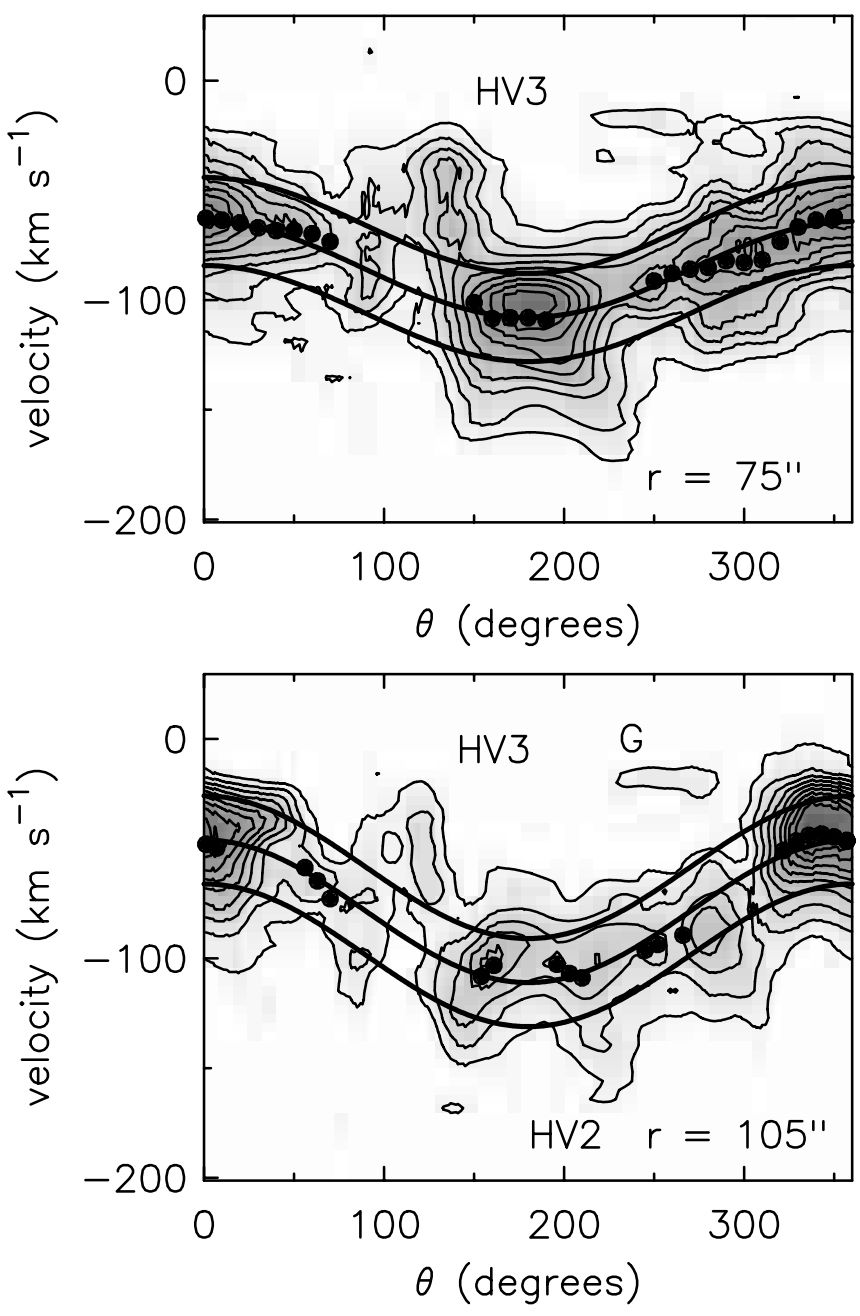

Fig. 6. continued.

26 dwarf galaxies, observed at $27^{\prime \prime}$ resolution, we find a mean HI velocity dispersion of $9.5 \pm 0.4 \mathrm{~km} \mathrm{~s}^{-1}$ (Stil \& Israel 2001). Thus, the velocity dispersion of the gas in NGC 1569, not even considering the various clouds with even higher discrepant velocities, already exceeds that of less disturbed galaxies by more than a factor of two. Examples of single-beam line profiles, sampling local conditions in NGC 1569 at 13 ". 5 resolution are shown in Fig. 9. The contribution of rotation to the width of these profiles is negligible and the broad profiles can often be fitted with a single Gaussian component (Figs. 9A, 9B). Such large velocity dispersions cannot be thermal, as they would then imply temperatures $\sim 5 \times 10^{4} \mathrm{~K}$ characteristic of ionized rather than neutral hydrogen. Thus, these broad line profiles must indicate turbulent velocities greatly exceeding those in other galaxies. At some places the line profile is clearly resolved into multiple components (Fig. 9C) - note that the profiles in 9B and 9C are separated by only $15^{\prime \prime}$ in right ascension. Locations of more modest velocity dispersions $\sim 8 \mathrm{~km} \mathrm{~s}^{-1}$ can only occasionally be identified.

No attempt was made to fit a mass model to the rotation curve of NGC 1569, because the asymmetric drift correction introduces a large uncertainty. However, a model of the rotation curve based on the visible mass (stars and gas) provides
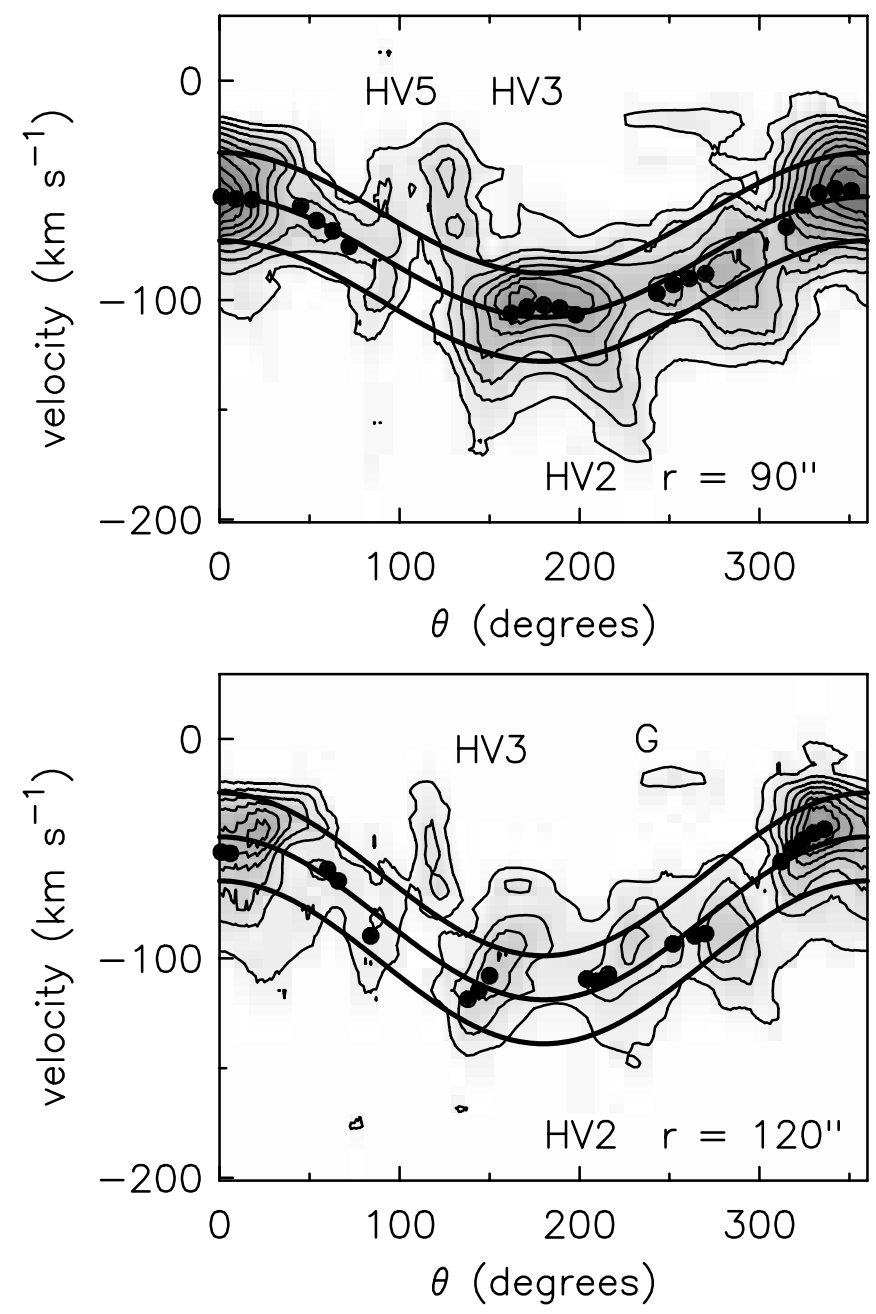

a useful reference frame for the observed rotation curve and the high velocity HI. In Fig. 10 we present model rotation curves for the observable mass components of NGC 1569 in comparison with the observed curve.

We assumed vertical mass distributions for the stars and the HI folowing a $\operatorname{sech}^{2}\left(z / z_{0}\right)$ law with scaleheight $z_{0}=0.3 \mathrm{kpc}$. Flatter mass distributions correspond to steeper rises of the rotation curve. We normalized the azimuthally averaged HI column-density profile to a total mass of $1.7 \times 10^{8} M_{\odot}$ to account for primordial helium. The gaseous mass does not include $\sim 2 \times 10^{6} M_{\odot}$ molecular gas from the bright starformation region west of starcluster A (Greve et al. 1996). We scaled the $R$-band surface brightness profile to an integrated extinctioncorrected magnitude $R=9.19(R=10.69$ magnitude from NED and extinction correction in $R$ based on $E(B-V)=0.56$ from Israel 1988). We have neglected any nebular emission line contamination of $R$-band intensities, as the contribution of the $\mathrm{H} \alpha$ line to $R$-band emission is typically only $10 \%$ to $20 \%$ for an equivalent width of $14.5 \mathrm{~nm}$ (Kennicutt \& Kent 1983).

The model HI rotation curve is comparable to observed rotational velocities. Inclusion of the stellar rotation curve produces a discrepancy between the observed and the predicted rotation curve for $\left(M / L_{R}\right)_{*}>0.02$ (Fig. 10). Stellar population 

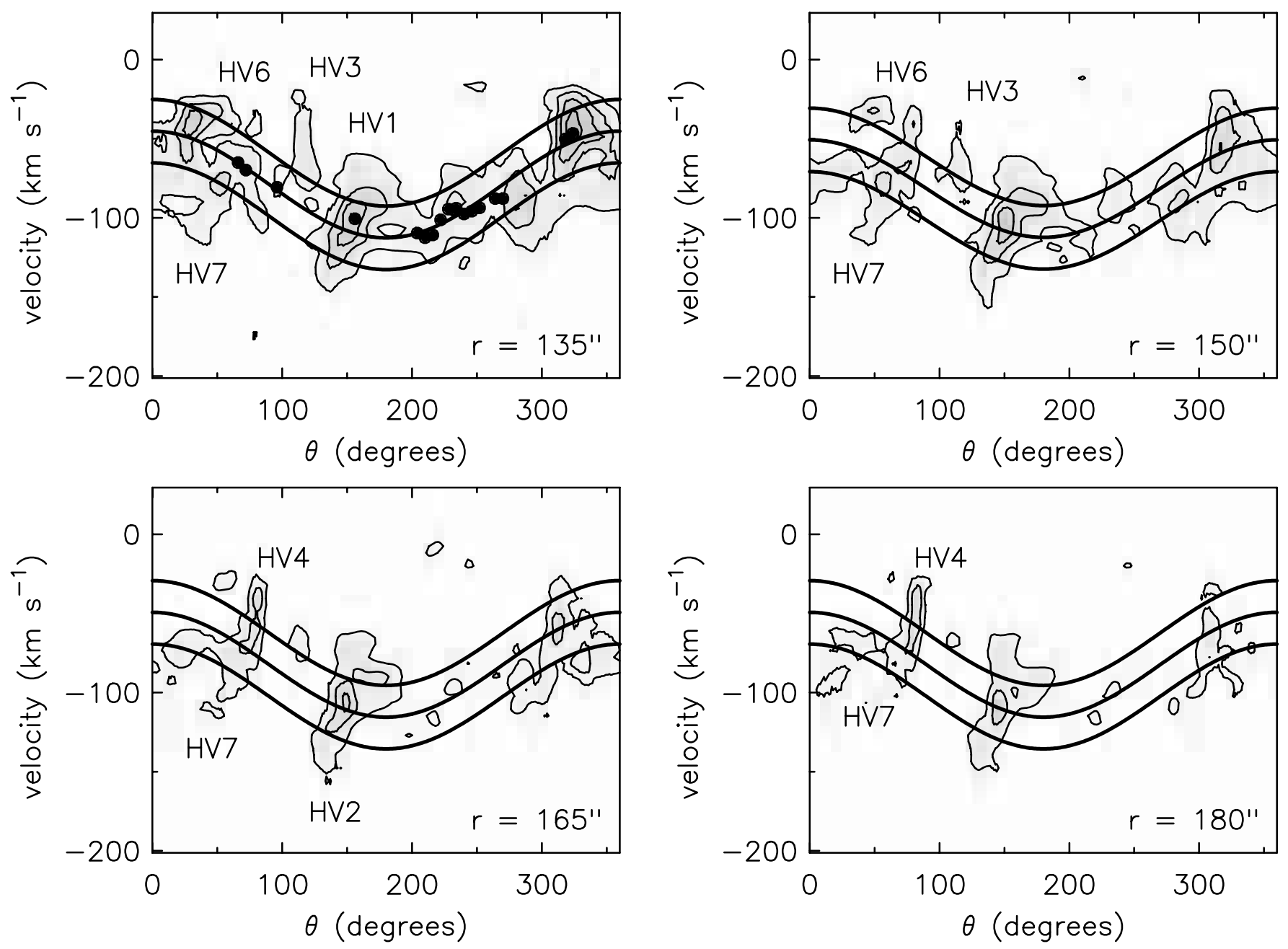

Fig. 6. continued.

synthesis models with $Z=0.004$ and steady star formation rates $1 M_{\odot} \mathrm{yr}^{-1}$ predict $\left(M / L_{R}\right)_{*}$ between 0.05 and 0.1 . after $10^{8} \mathrm{yr}$, depending on the assumed stellar initial mass function (Leitherer et al. 1999). The difference between these models and the observed rotational velocities imply that turbulent motions dominate the inner parts.

\subsection{The Western HI Arm}

The Western HI Arm appears as an extension of the highcolumn-density ridge in the HI column density map (Fig. 3) between $\alpha=4^{\mathrm{h}} 25^{\mathrm{m}} 12^{\mathrm{s}}$ and $\alpha=4^{\mathrm{h}} 25^{\mathrm{m}} 50^{\mathrm{s}}, \delta \approx 64^{\circ} 44^{\prime}$. It is much more extended than the system of $\mathrm{H} \alpha$ filaments of NGC 1569. Kinematically, it stands out because it is redshifted by about $20 \mathrm{~km} \mathrm{~s}^{-1}$ from its surroundings (Figs. 4-6). On its eastern side, emission of the Arm blends into emission from the main body of NGC 1569 .

It seems unlikely that the Western HI Arm is the symmetric counterpart of NGC 1569-HI and the HI bridge. The Western HI Arm is significantly closer to the optical center of NGC 1569 than NGC 1569-HI and the HI bridge. Furthermore, it has a prograde velocity with respect to the rotation of NGC 1569, as opposed to the apparent retrograde velocity of NGC 1569-HI (Stil \& Israel 1998).

\subsection{Neutral high-velocity gas}

Figure 6 amply illustrates the presence of local but large departures from ordered rotation in NGC 1569. Attempts to separate high-velocity gas from the general gas mass by fitting multiple Gaussians to local line profiles do not lead to satisfactory results because of insuffiencient signal-to-noise ratios outside the HI ridge, complex line profile shapes and confusion with Galactic HI. Instead, we have isolated the high-velocity gas by subtracting a model for the regularly rotating gas from the data. To this end, we first constructed a model velocity field from the rotation curve in Table 1 . In reasonable agreement with the data in Fig. 6, we assumed rotational velocities $v(r) \sin i=32.5 \mathrm{~km} \mathrm{~s}^{-1}$ at radii $r \geq 120^{\prime \prime}$. The model was required to have line-of-sight velocities prescribed by the rotation curve and velocity dispersions equal to the mean dispersion $\langle\sigma\rangle=21.3 \mathrm{~km} \mathrm{~s}^{-1}$. Model line profile amplitudes are equal to the observed line intensity at the velocity predicted by the model rotation curve. We then constructed HI columndensity maps of the residual emission by adding the channel maps at both positive and negative velocities with respect to the model rotation velocity. These residual HI column-density maps are shown as contours over the $\mathrm{H} \alpha$ image in Fig. 11. In order to suppress noise from regions without significant residual 
Table 1. The rotation curve of NGC 1569.

\begin{tabular}{cccccc}
\hline \hline $\begin{array}{c}r \\
{[1]}\end{array}$ & $\begin{array}{c}v_{\text {sys }} \\
{[2]}\end{array}$ & $\begin{array}{c}v(r) \sin i \\
{[3]}\end{array}$ & $\begin{array}{c}v_{\text {rec }}(r) \sin i \\
{[4]}\end{array}$ & $\begin{array}{c}v_{\text {app }}(r) \sin i \\
{[5]}\end{array}$ & $\begin{array}{c}\sigma_{\mathrm{v}} \\
{[6]}\end{array}$ \\
$\operatorname{arcsec}$ & $\mathrm{km} \mathrm{s}^{-1}$ & $\mathrm{~km} \mathrm{~s}^{-1}$ & $\mathrm{~km} \mathrm{~s}^{-1}$ & $\mathrm{~km} \mathrm{~s}^{-1}$ & $\mathrm{~km} \mathrm{~s}^{-1}$ \\
\hline 15 & $-78.3 \pm 0.8$ & $0.4 \pm 1.1$ & $-1.9 \pm 1.3$ & $7.9 \pm 2.4$ & $20.2 \pm 1.0$ \\
30 & $-78.4 \pm 1.5$ & $-0.5 \pm 2.3$ & $-5.0 \pm 6.7$ & $7.3 \pm 5.7$ & $20.6 \pm 1.4$ \\
45 & $-81.2 \pm 1.1$ & $7.1 \pm 1.6$ & $4.2 \pm 6.2$ & $11.5 \pm 5.4$ & $21.6 \pm 3.1$ \\
60 & $-92.1 \pm 0.8$ & $17.7 \pm 1.0$ & $10.4 \pm 1.9$ & $22.3 \pm 3.5$ & $21.6 \pm 3.4$ \\
75 & $-85.2 \pm 0.8$ & $21.8 \pm 1.1$ & $25.2 \pm 4.2$ & $25.3 \pm 2.3$ & $22.6 \pm 4.3$ \\
90 & $-79.5 \pm 1.0$ & $27.4 \pm 1.3$ & $34.0 \pm 3.8$ & $16.6 \pm 2.1$ & $20.8 \pm 3.4$ \\
105 & $-77.7 \pm 1.0$ & $32.4 \pm 1.3$ & $37.6 \pm 4.3$ & $19.0 \pm 3.1$ & $21.1 \pm 4.1$ \\
120 & $-80.9 \pm 1.4$ & $37.0 \pm 2.0$ & $43.4 \pm 7.8$ & $27.7 \pm 4.9$ & $22.0 \pm 3.0$ \\
135 & $-78.1 \pm 1.4$ & $33.6 \pm 2.2$ & $45.5 \pm 5.0$ & $25.5 \pm 4.3$ & $21.2 \pm 3.8$ \\
150 & $-80.7 \pm 1.8$ & $30.7 \pm 3.3$ & $31.3 \pm 12$ & $32.9 \pm 5.5$ & - \\
180 & $-85.7 \pm 1.2$ & $24.6 \pm 1.9$ & - & - & - \\
\hline
\end{tabular}

Column definitions: [1] radius in arcseconds; [2] systemic velocity in $\mathrm{km} \mathrm{s}^{-1}$; [3] rotation velocity both sides in $\mathrm{km} \mathrm{s}^{-1}$ (not corrected for inclination); [4] rotation velocity receding side in $\mathrm{km} \mathrm{s}^{-1}$; [5] rotation velocity approaching side in $\mathrm{km} \mathrm{s}^{-1}$; [6] mean velocity dispersion in $\mathrm{km} \mathrm{s}^{-1}$ and rms scatter around the mean. Notes: the fit of the receding side at $r=45^{\prime \prime}$ was abandoned because of incoherent fluctuations of the data; The inconsistency between approaching and receding side at $r=60^{\prime \prime}$ is due to the velocities in the interval $300^{\circ}<\theta<40^{\circ}$.

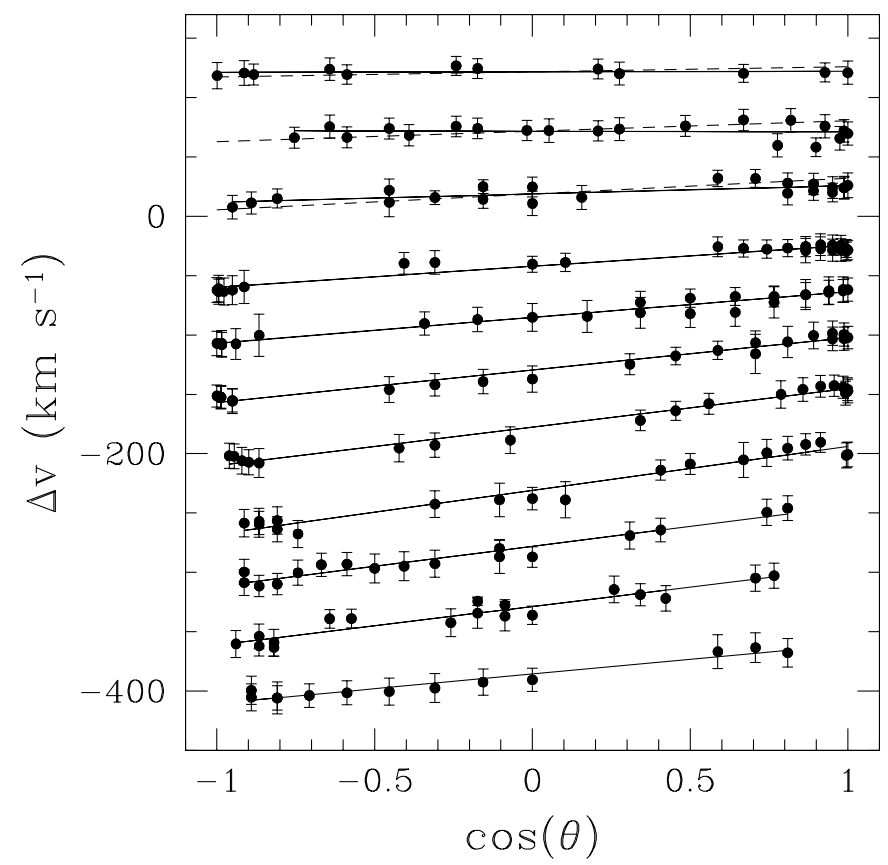

Fig. 7. Fits to $v_{\mathrm{obs}}=v(r) \sin (i) \cos (\theta)$. From top to bottom $r=15^{\prime \prime}$ to $r=150^{\prime \prime}$ at $15^{\prime \prime}$ intervals and $r=180^{\prime \prime}$ at the bottom. For clarity, data for different radii were shifted by $50 \mathrm{~km} \mathrm{~s}^{-1}$ relative to the data at $r=75^{\prime \prime}$. The errorbars indicate half the fitted velocity dispersion as justified in the text. The dashed lines indicate the relation expected for solid body rotation within $r=60^{\prime \prime}$.

emission, only positive and negative residuals stronger than $2 \sigma$ in three consecutive velocity channels were included in the summation. Line profiles of the residual components are shown in Fig. 12.

The validity of these residuals as high-velocity components was verified in the channel maps and in Fig. 6. The close correspondence between Fig. 11 and peculiar structure in the channel maps adds to our confidence in the residuals as distinct and separate features (see also Fig. 13). Only the residuals

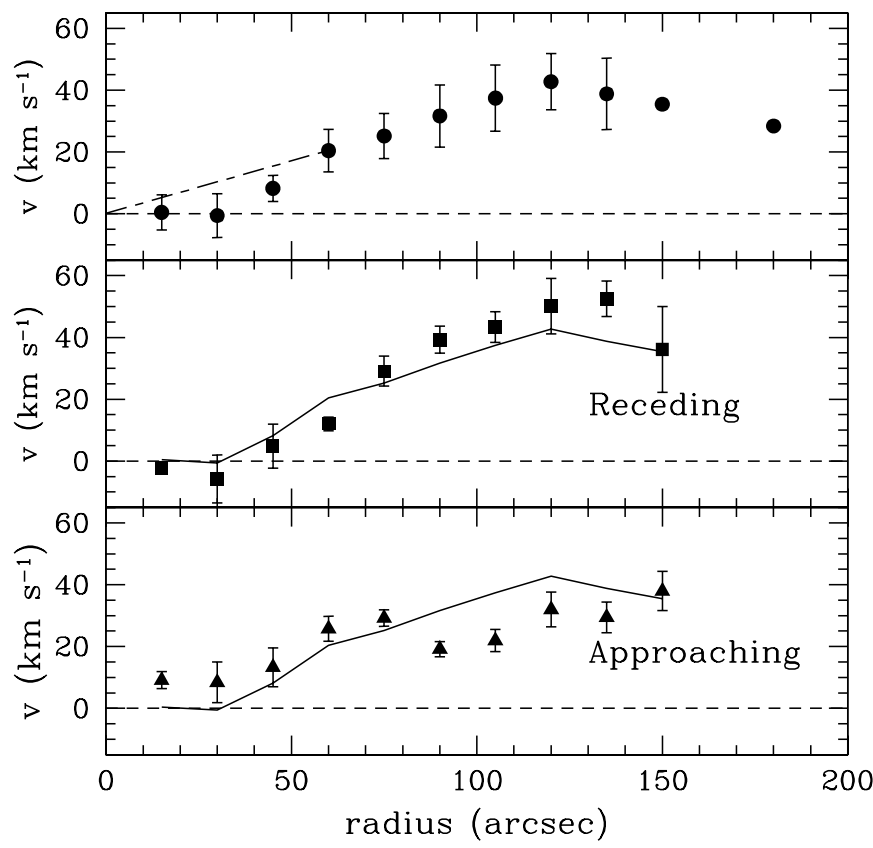

Fig. 8. The rotation curve of NGC 1569 fitted to both sides simultaneously (upper panel) and to the approaching and receding sides separately (lower panels). The errorbars in the upper panel indicate the difference between the one-sided fits (except for $r=75^{\prime \prime}$, where the mean of the errors at $60^{\prime \prime}$ and $90^{\prime \prime}$ is used). The dot-dashed line in the upper panel indicates solid-body rotation within $60^{\prime \prime}$ from the center. The solid line in the lower panels is the two-sided rotation curve from the upper panel. The velocity at radii $r \leq 60^{\prime \prime}$ was measured from the $13^{\prime \prime}$ resolution data, and the point at $r=180^{\prime \prime}$ was measured from the $60^{\prime \prime}$ resolution data. Intermediate points were measured from the $27^{\prime \prime}$ resolution data.

shown in panels 6 and 8 should be considered as the spurious residuals of bright emission with a small peculiar velocity at $r=135^{\prime \prime}, \theta=280^{\circ}$ (residual 6) and $r=120^{\prime \prime}, \theta=325^{\circ}$ (residual 8), and not as separate components. Component HV1 is associated with the Western HI Arm, which is redshifted 

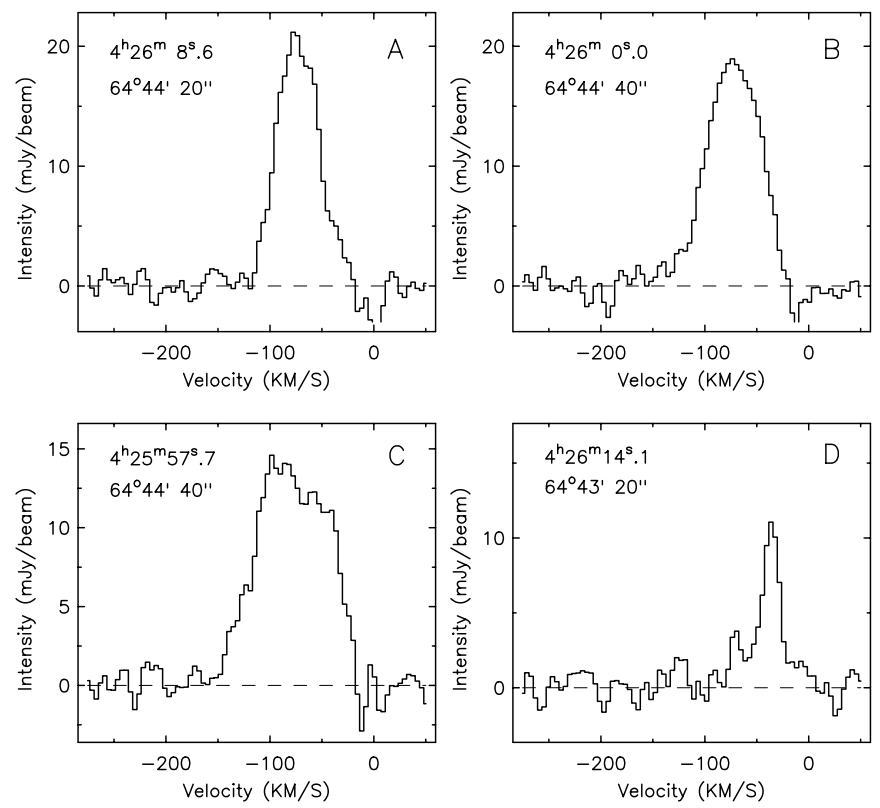

Fig. 9. Examples of single-beam line profiles from NGC 1569. Right ascension and declination are given in the upper left corner of each panel. Panels a) and b) show profiles that can be represented by a single broad Gaussian. Panel c) shows a line of sight with two components with a velocity difference of $46 \mathrm{~km} \mathrm{~s}^{-1}$. Panel d) shows a line profile with a dispersion of $9 \mathrm{~km} \mathrm{~s}^{-1}$, which is observed in most galaxies but is uncommon in NGC 1569.
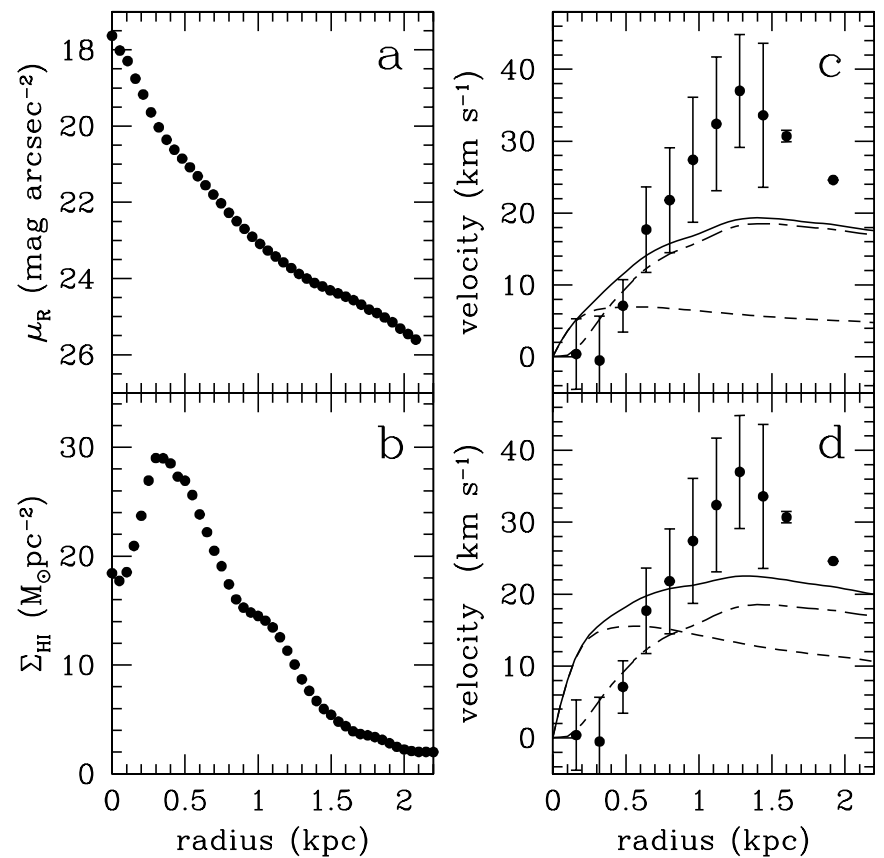

Fig. 10. Model rotation curves for NGC 1569. a) $R$-band surface brightness profile of NGC 1569. Foreground stars were masked out; b) gas surface density, taking into account primordial helium; c) observed rotation curve compared with model rotation curves for the stars with $\left(M / L_{R}\right)_{*}=0.02 M_{\odot} / L_{R \odot}$ (short dashed), gas (long/short dashed) and stars+gas (continuous line); d) as in c), but with $\left(M / L_{R}\right)_{*}=0.1 M_{\odot} / L_{R \odot}$.
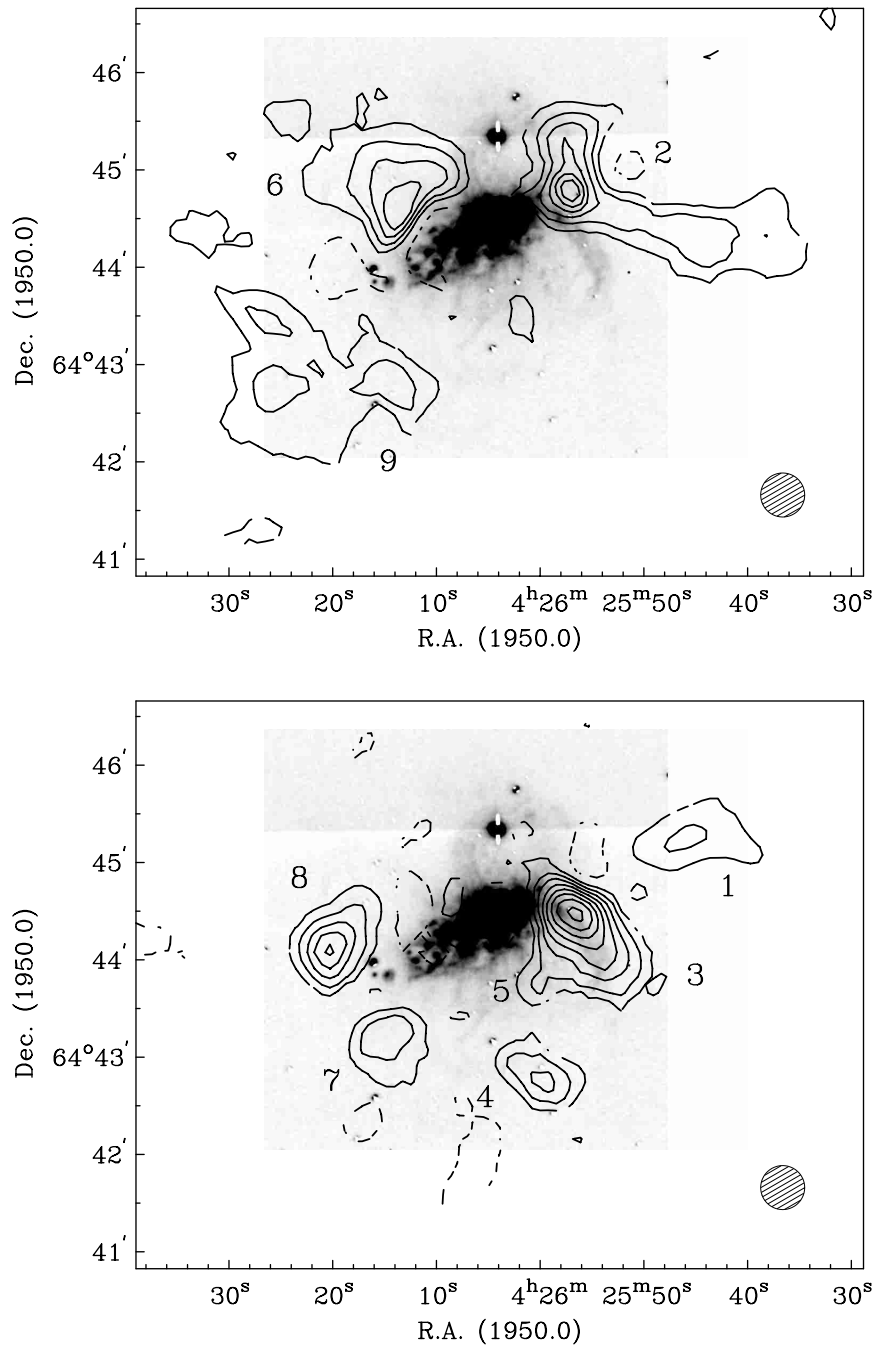

Fig. 11. Distribution of the high-velocity HI gas in NGC 1569 in relation to the $\mathrm{H} \alpha$ emission. Contours are at $N_{\mathrm{HI}}=-2$ (dashed), 2, 4, $6, \ldots \times 10^{20} \mathrm{~cm}^{-2}$. Components with a negative (approaching) velocity relative to the model rotation velocity are shown in the top panel. The bottom panel shows emission with a positive (receding) velocity relative to the rotation of NGC 1569 . Numbers refer to the first column of Table 2. Features 6 and 8 are not considered as real and separate high-velocity clouds (see text).

with respect to its surroundings (Fig. 4). HV2 is identified as the brightest emission in the channel maps at velocities $v_{\text {hel }}=-168 \mathrm{~km} \mathrm{~s}^{-1}$ to $v_{\text {hel }}=-135 \mathrm{~km} \mathrm{~s}^{-1}$. Its remarkable extension to the southwest is real and clearly visible also in the channel maps and in Fig. 6 out to radius $r=180^{\prime \prime}$. HV2 is just northeast of the $\mathrm{H} \alpha$ arm and west of the bubble in the north of NGC 1569 designated Arc 1 by Tomita et al. (1994) and complex B by Martin (1998).

Components HV3 and HV4 are visible in the channel maps around $v_{\text {hel }}=-53 \mathrm{~km} \mathrm{~s}^{-1}$ and in the 13. '.5 resolution channel map at $v_{\text {hel }}=-48.8 \mathrm{~km} \mathrm{~s}^{-1}$ (Fig. 13). The structure of HV3 is remarkably similar to that of the $\mathrm{H} \alpha$ arm: $\mathrm{HI}$ and $\mathrm{H} \alpha$ emission have similar extent and display the same curvature. The $\mathrm{H} \alpha$ emission is only marginally offset to the south of HV3 by $\sim 5^{\prime \prime}$. However, their velocities are very different. HV3 is redshifted relative to the systemic velocity of NGC 1569 , 

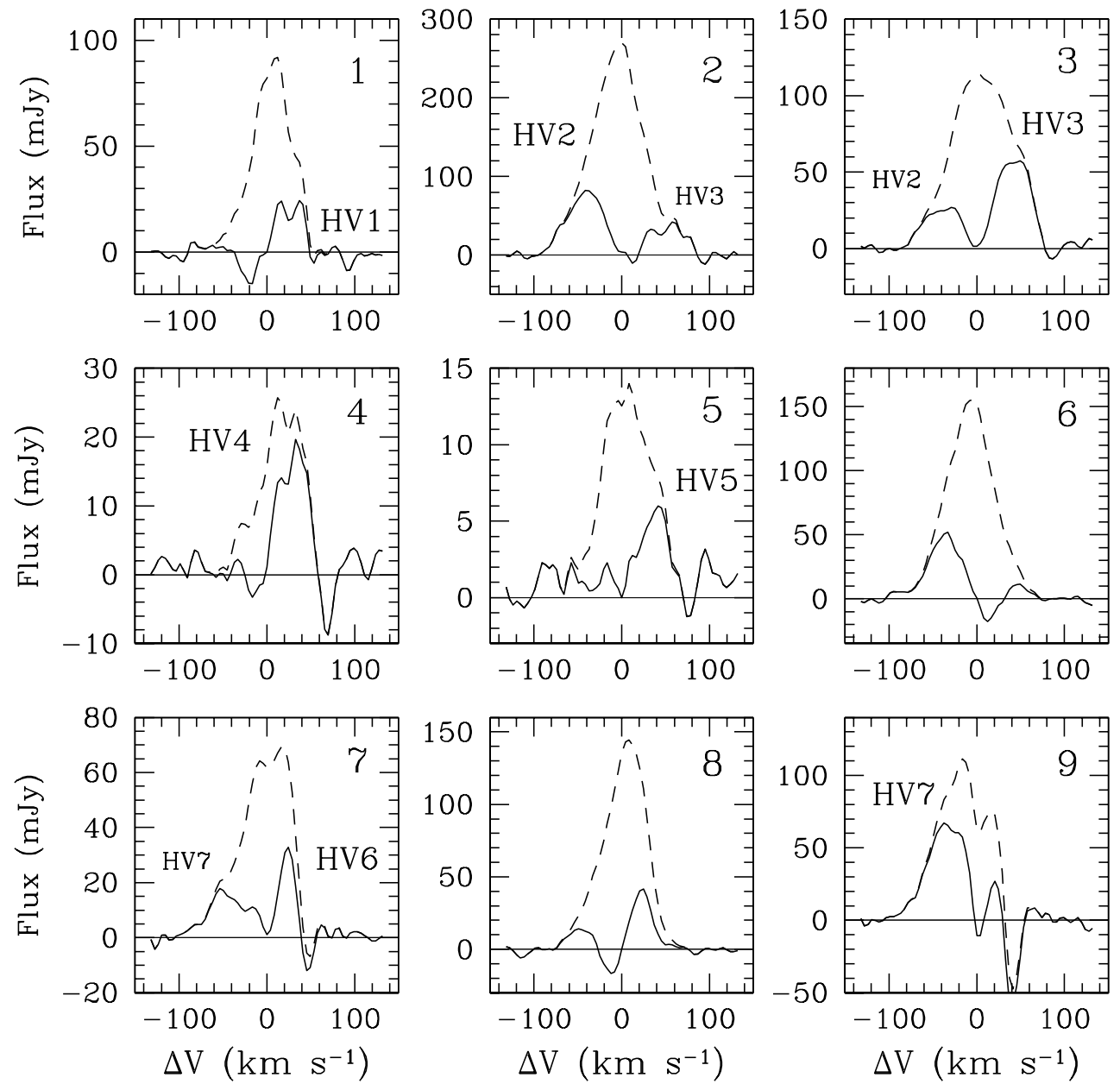

Fig. 12. Line profiles of high-velocity components in NGC 1569. The dashed line is the line profile before model-subtraction. Solid lines are the residual line profiles. The zero-point of the velocity axis is the model rotation velocity. High velocity components indicated as HV1 through HV7 can be identified in Fig. 6. The panel numbers refer to column [1] in Table 2.

whereas the $\mathrm{H} \alpha$ arm is blueshifted by approximately $40 \mathrm{~km} \mathrm{~s}^{-1}$ (Tomita et al. 1994) with respect to the systemic velocity. HV5 is separate from HV3, as clearly shown in Fig. 13. HV6 may be related to HV4; both are found in the direction of Waller's Arc 1 and together may be its neutral counterpart. The emission seen in Fig. 3 (top) at this location is in fact almost entirely due to HV4 and HV 6. The location and velocity of HV7 suggest that it is associated with the HI bridge between NGC 1569 and NGC 1569-HI.

The parameters of the high-velocity components are listed in Table 2. These components account for approximately $10 \%$ of the HI mass of NGC 1569. Their mean velocity, weighted by mass, is $-91 \mathrm{~km} \mathrm{~s}^{-1}$, effectively indistinguishable from the systemic velocity of NGC 1569 . This is to be expected if the gas was expelled from the galaxy, for instance by the starburst. We estimate a lower limit to the kinetic energy of a highvelocity component in the restframe of NGC 1569 by $E_{\mathrm{k} ; \min }=$ $\frac{1}{2} M_{\mathrm{HI}}\left(v-v_{\text {sys }}\right)^{2}$, where $v$ and $v_{\text {sys }}$ are the observed heliocentric velocity of the cloud and of NGC 1569 respectively. These energies are included in Table 2 . The minimal kinetic energy in the high-velocity components is $2.5 \times 10^{46} \mathrm{~J}$. This is $0.3 \%$ of the mechanical energy estimated by Heckman et al. (1995) to be released in the starburst. However, the mass of the neutral high-velocity gas is up to an order of magnitude larger than the combined mass of the X-ray gas and the $\mathrm{H} \alpha$ filaments (Martin 1999 and references therein).

The possible association of HV1 and HV4 with $\mathrm{H} \alpha$ filaments raises the question whether the high-velocity components are neutral gas at the edges of a large bubble as was suggested for the $\mathrm{H} \alpha$ filaments (e.g. Waller 1991; Tomita et al. 1994). We looked for evidence of such a bubble in the form of doppler ellipses combining several high-velocity components. The position-velocity diagram in Fig. (14) intersects high-velocity components HV2, HV3, HV4, and HV5. A candidate doppler ellipse is the region between HV4 and HV5. However, its morphology is quite chaotic. The faint emission at the extreme velocities is separated by $70 \mathrm{~km} \mathrm{~s}^{-1}$. If this emission is interpreted as representing the approaching and receding edges of an expanding bubble, the implied expansion velocity of $35 \mathrm{~km} \mathrm{~s}^{-1}$ is three times less than expansion speeds observed in $\mathrm{H} \alpha$ (Tomita et al. 1994; Heckman et al. 1995; Martin 1998). The velocity coverage of the present HI observations $\left(-296 \mathrm{~km} \mathrm{~s}^{-1}\right.$ to $\left.+116 \mathrm{~km} \mathrm{~s}^{-1}\right)$ includes most of the observed velocities of the $\mathrm{H} \alpha$ filaments (cf. Heckman et al. 1995; 
Table 2. High velocity components identified in NGC 1569.

\begin{tabular}{|c|c|c|c|c|c|c|c|}
\hline $\begin{array}{c}\text { Panel } \\
{[1]}\end{array}$ & $\begin{array}{c}\text { Name } \\
{[2]}\end{array}$ & $\begin{array}{c}\alpha_{1950} \\
{[3]}\end{array}$ & $\begin{array}{c}\delta_{1950} \\
{[4]}\end{array}$ & $\begin{array}{c}M_{\mathrm{HI}} \\
{[5]} \\
10^{6} M_{\odot} \\
\end{array}$ & $\begin{array}{r}v_{\text {hel }} \\
{[6]} \\
\mathrm{km} \mathrm{s}^{-1} \\
\end{array}$ & $\begin{array}{r}\Delta v \\
{[7]} \\
\mathrm{km} \mathrm{s}^{-1} \\
\end{array}$ & $\begin{array}{c}\log \left(E_{\mathrm{k}}\right) \\
{[8]} \\
\mathrm{J} \\
\end{array}$ \\
\hline 1 & HV1 & $4^{\mathrm{h}} 25^{\mathrm{m}} 45^{\mathrm{s}} .5$ & $64^{\circ} 45^{\prime} 15^{\prime \prime}$ & $0.4 \pm 0.1$ & -68 & 37 & 43.6 \\
\hline 2 & HV2 & $4^{\mathrm{h}} 25^{\mathrm{m}} 56^{\mathrm{s}} .9$ & $64^{\circ} 44^{\prime} 45^{\prime \prime}$ & $4.6 \pm 0.2$ & -139 & -43 & 46.2 \\
\hline 3 & HV3 & $4^{\mathrm{h}} 25^{\mathrm{m}} 56^{\mathrm{s}} .9$ & $64^{\circ} 44^{\prime} 30^{\prime \prime}$ & $3.1 \pm 0.2$ & -51 & 41 & 45.3 \\
\hline 4 & HV4 & $4^{\mathrm{h}} 25^{\mathrm{m}} 59^{\mathrm{s}} .2$ & $64^{\circ} 42^{\prime} 45^{\prime \prime}$ & $0.81 \pm 0.01$ & -47 & 29 & 44.9 \\
\hline 5 & HV5 & $4^{\mathrm{h}} 26^{\mathrm{m}} 00^{\mathrm{s}} \cdot 0$ & $64^{\circ} 43^{\prime} 45^{\prime \prime}$ & $0.26 \pm 0.09$ & -48 & 35 & 44.4 \\
\hline 6 & & $4^{\mathrm{h}} 26^{\mathrm{m}} 13^{\mathrm{s}} .7$ & $64^{\circ} 44^{\prime} 37^{\prime \prime}$ & & & & \\
\hline 7 & HV6 & $4^{\mathrm{h}} 26^{\mathrm{m}} 15^{\mathrm{s}} .2$ & $64^{\circ} 43^{\prime} 15^{\prime \prime}$ & $0.76 \pm 0.32$ & -35 & 22 & 45.1 \\
\hline 8 & & $4^{\mathrm{h}} 26^{\mathrm{m}} 20^{\mathrm{s}} 3$ & $64^{\circ} 44^{\prime} 05^{\prime \prime}$ & & & & \\
\hline 9 & HV7 & $4^{\mathrm{h}} 26^{\mathrm{m}} 21^{\mathrm{s}} .8$ & $64^{\circ} 42^{\prime} 45^{\prime \prime}$ & $4.0 \pm 0.4$ & -92 & -38 & 44.9 \\
\hline Total & & & & 13.9 & -91 & & 46.4 \\
\hline
\end{tabular}

Column definitions: [1] Identification panel in Fig. 12; [2] designation for residuals considered genuine high-velocity components [3] right ascension (1950) of maximum HI column density; [4] declination (1950) of maximum HI column density; [5] HI mass assuming a distance of $2.2 \mathrm{Mpc}$; [6] heliocentric velocity; [7] deviation in line of sight velocity from rotation model; [8] Kinetic energy.

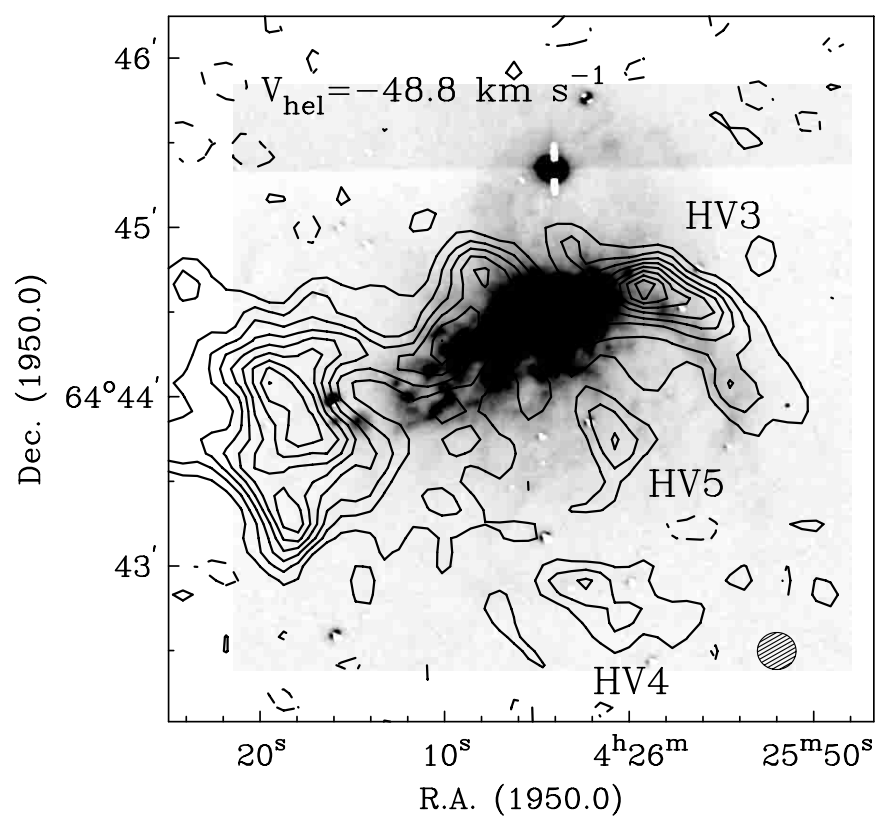

Fig. 13. The $13^{\prime \prime}$ resolution channel map at $v_{\text {hel }}=-48.8 \mathrm{~km} \mathrm{~s}^{-1}$ as contours over the $\mathrm{H} \alpha$ emission (grayscale). The velocity of the channel map is near the central velocity of high-velocity components HV3, HV4 and HV5. Contours are at $-1.94,1.94(2 \sigma), 3.88,5.82, \ldots$ $\mathrm{mJy} /$ beam. At this velocity, $\mathrm{HI}$ in regular rotation is expected to be limited to the area east of the optical galaxy. The spatial coincidence of $\mathrm{HV} 3$ and the $\mathrm{H} \alpha$ arm is clearly visible. The structure obvious in this channel map has a clear counterpart in the velocity field depicted in Fig. 4.

Fig. 4). Also, the clumpy ring in Fig. 14 is even less obvious in $\mathrm{p}-\mathrm{V}$ maps at different position angles. We have thus not found any conclusive evidence for a large bubble associated with the high-velocity HI.

Emission from the HI bridge appears to the left (southsoutheast) of HV4 in Fig. 14. There is no trace of the HI bridge nearer to NGC 1569 than the position of HV4. If the HI bridge does not terminate at this location, HV4 is the obvious

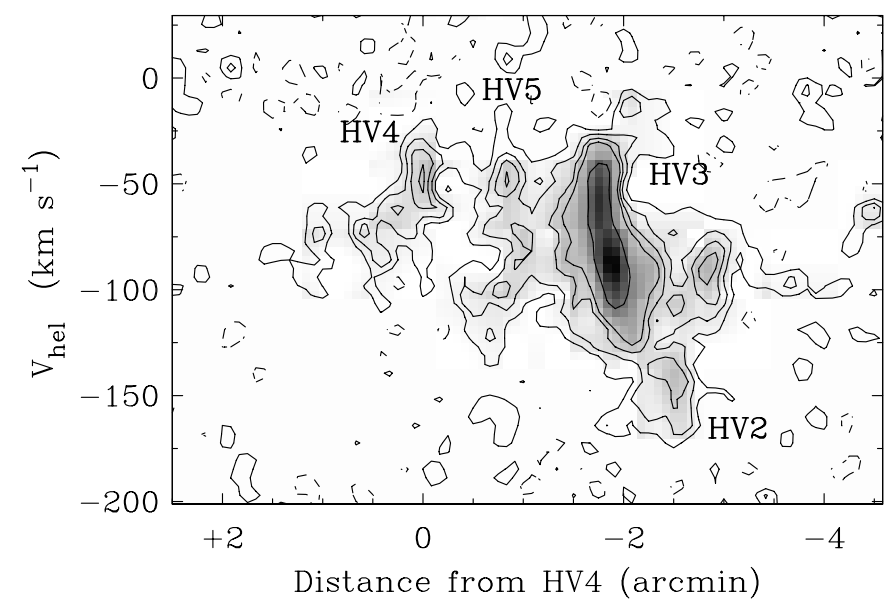

Fig. 14. Position-velocity map of the $13^{\prime \prime}$ resolution data along a line through high-velocity components HV3, HV4 and HV5. The only candidate doppler-ellipse is the region between HV4 and HV5. This area coincides with complex G in Martin (1998), which expands with a velocity of $64 \mathrm{~km} \mathrm{~s}^{-1}$. Emission to the left of HV4 (south-southeast) is associated with the $\mathrm{HI}$ bridge. Contours are at $-1.45,1.45(1.5 \sigma)$, $2.90,4.35,5.80,11.60$ and $23.20 \mathrm{mJy}$ per beam.

candidate for the continuation of bridge emission in the line of sight towards NGC 1569. It is interesting to compare the velocity of HV4 with the velocity of $\mathrm{H} \alpha$ Arc 1 . A long-slit spectrum in position angle $160^{\circ}$ procured by Heckman et al. (1995) intersects $\mathrm{H} \alpha$ Arc 1 somewhat to the east of the $\mathrm{p}-\mathrm{V}$ map in Fig. 14. If $\mathrm{H} \alpha$ Arc 1 is the brightest velocity component between $89^{\prime \prime}$ and $97^{\prime \prime}$ southeast of starcluster A in that spectrum, its velocity of $-75 \pm 5 \mathrm{~km} \mathrm{~s}^{-1}$ is very similar to that of the HI gas southeast of HV4.

\section{Discussion}

\subsection{The rotation curve}

The overall HI kinematics of NGC 1569 are those of a global velocity gradient along the major axis of the galaxy (Fig. 4). 
This velocity gradient must be caused by rotational movement. Were it caused by expansion, we would expect the velocity gradient to be along the minor axis, even for radial expansion in the plane of the disk. The rotation curve of NGC 1569 differs from that of other dwarf galaxies as its inner velocities are much lower than would be expected from an extrapolation of the rotation at larger radii. The region of low velocities, in effect of zero rotation, coincides with the location of the starburst, and in fact with most of the optically visible galaxy. Thus, turbulent motions initiated by the starburst appear to dominate the inner HI disk remnant. The absence of any significant decrease in the HI velocity dispersion near $r=60^{\prime \prime}$ indicates that the ISM is also stirred up in the outer regions of the galaxy, with a ratio of rotational to random velocities of about two. Although the starburst did not initiate a global blow-away of the interstellar medium from NGC 1569, it appears to have left its imprint on the outer disk. This confirms, at least for NGC 1569, the conclusions reached by Mac Low \& Ferrara (1999) from hydrodynamic simulations. Only a partial blow-out seems to occur, even for gas circular velocities of only $\sim 35 \mathrm{~km} \mathrm{~s}^{-1}$.

The rotation curve of NGC 1569 flattens at 90" (1 kpc) from the center, with amplitude $v_{\text {flat }}=35 \pm 6 \mathrm{~km} \mathrm{~s}^{-1}$ for an inclination of $60^{\circ}$. We attribute the apparent slight decline of the rotation curve beyond 120" (also observed by Reakes 1980), to the peculiar velocities of the HI bridge on the receding side and the Western HI Arm on the approaching side. Could the apparent decline be caused by warping of the HI disk? The kinematic signatures of a warped disk are systematic changes in the position angle of the kinematic major axis (dependending on the orientation of the line of nodes and the inclination), and changes in the observed rotational velocity due to changing inclination. A change in position angle of the kinematic major axis would appear as a phase shift of the cosine shape in Fig. 6. There is no evidence for such a phase shift, from which we deduce an upper limit to a change in position angle of $20^{\circ}$.

\subsection{Neutral high-velocity gas}

Relatively abundant (i.e. $10 \%$ by mass) high-velocity gas has now been observed in NGC 1569 in both $\mathrm{H} \alpha$ and HI- $21 \mathrm{~cm}$ emission. The associated HI and HII peculiar velocities are typically 30 to $50 \mathrm{~km} \mathrm{~s}^{-1}$ (e.g. Tomita et al. 1994; Heckman et al. 1995, and Sect. 3.7). Doppler ellipses observed in the $\mathrm{H} \alpha$ filaments along the minor axis indicate expansion velocities of about $100 \mathrm{~km} \mathrm{~s}^{-1}$ (Heckman et al. 1995; Martin 1998), more than twice the local circular velocity. Such large expansion velocities that far from the center suggest that at least part of the ionized gas associated with the $\mathrm{H} \alpha$ filaments may escape from NGC 1569 (Martin 1998). The temperature of the hot gas is also greatly exceeds the limit for escape from NGC 1569 (Della Ceca et al. 1996). How does the neutral high-velocity gas relate to the galactic wind from NGC 1569? This question has important implications for the ability of the galactic wind to remove chemically enriched material from NGC 1569 permanently, and the escape of ionizing photons from the galaxy.

Problems arise if all or most of the high-velocity $\mathrm{HI}$ is attributed to galactic winds blowing out of NGC 1569. The mass of the cold high-velocity HI components exceeds that of the hot ionized gas by up to an order of magnitude. This would imply that the gas blown out of NGC 1569 is largely neutral, contrary to what is expected. The cooling time of the hot X-ray gas is of the order of $10^{9}$ years, but this timescale relates to the hot interior, not the bubble walls (Heckman et al. 1995). The mass discrepancy is alleviated somewhat if a fraction of the highvelocity HI should be part of the HI bridge, the amount of neutral gas to be associated with the galactic wind is correspondingly smaller. In a previous paper (Stil \& Israel 1998) we have argued that NGC 1569-HI and the HI bridge are unlikely to be related to the outflow from NGC 1569.

The strongest observational evidence linking neutral highvelocity gas with the outflow, are the apparent associations of $\mathrm{H} \alpha$ Arc 1 (Waller 1991) with high-velocity components HV4/HV6 and the $\mathrm{H} \alpha$ arm with HV3. The $\mathrm{H} \alpha$ arm is often interpreted as the inner edge of a bubble on the southern side of NGC 1569 (Waller 1991; Martin 1998). However, in contrast to the spatial coincidence of the $\mathrm{H} \alpha$ arm and $\mathrm{HV} 3$, their radial velocities are very different. This is a significant objection because simple radial outflow models cannot simultaneously explain blueshifted ionized gas velocities and redshifted neutral gas velocities. If both participate in a radial outflow, they should either both be blueshifted, or both be redshifted with respect to the systemic velocity. On the other hand, if the $\mathrm{H} \alpha$ arm represents the inner edge of an expanding bubble, most of the expansion velocity will be in the plane of the sky, i.e. tangentially. For sufficiently large expansion velocities, a small asymmetry in the ionized and neutral gas distributions could lead to a significant difference in the (observed) radial velocities. Nevertheless, the observed radial velocity difference of $\sim 40 \mathrm{~km} \mathrm{~s}^{-1}$ is almost half the maximum expansion velocity inferred from the splitting of the $\mathrm{H} \alpha$ lines. Yet another possibility is that the dense, relatively cool bubble wall is unstable and moves into the hot wind which fills the bubble (Suchkov et al. 1994).

Alternatively, we could consider $\mathrm{HV} 3$ and the $\mathrm{H} \alpha$ arm to be part of the same gas system as the HI bridge, and to represent infall rather than outflow. This gas system would be exposed to the galactic winds. It might also be exposed directly to ionizing radiation from NGC 1569, as almost half of the ionizing flux produced inside the main body of the galaxy escapes (Israel 1988). Heckman et al. (1995) have argued that in the $\mathrm{H} \alpha$ filaments photo-ionization dominates over shock-heating. The $\mathrm{H} \alpha$ arm appears to be slightly displaced from HV3 towards the starburst region (Fig. 13). However, if the $\mathrm{H} \alpha$ arm is interpreted as the photo-ionized skin of a neutral gas cloud (HV3), the velocity difference between the neutral and ionized gas remains unexplained. Infalling gas colliding with the outflow would cause shocks that may provide an explanation for the detached X-ray emission observed by Heckman (1995) and Della Ceca et al. (1996) in the vicinity of the $\mathrm{H} \alpha$ arcs.

\section{Conclusions}

1) The $1.4 \mathrm{GHz}$ continuum flux of NGC 1569 is $438 \pm 5 \mathrm{mJy}$. Peaks in the radio continuum distribution coincide with the two 
brightest HII regions. The continuum emission largely follows the $\mathrm{H} \alpha$ distribution, including the so-called $\mathrm{H} \alpha$ arm.

2) The HI flux of NGC 1569 measured in the interferometer maps is $116 \mathrm{Jy} \mathrm{km} \mathrm{s}^{-1}$. This corresponds to an HI mass of $1.3 \times 10^{8} M_{\odot}$ at $2.2 \mathrm{Mpc}$. The HI structure of the galaxy is that of extended diffuse HI emission, centered on a clumpy ridge of dense gas associated with the small optical galaxy. Much further structure is evident, in the form of a counterrotating companion, and intervening bridge and other features of possible tidal origin.

3 ) There is no detectable rotation of the HI gas within radii of $60^{\prime \prime}(0.6 \mathrm{kpc})$ from the center, confirming earlier results obtained for the ionized gas by other authors. Beyond $0.6 \mathrm{kpc}$ from the center, the rotation velocity increases to $35 \pm 6 \mathrm{~km} \mathrm{~s}^{-1}$. An apparent turnover in the rotation curve is an artifact introduced by the presence of significant HI with peculiar velocities. Even excluding areas with high-velocity HI, NGC 1569 has a high average $\mathrm{HI}$ velocity dispersion of $\sigma_{\mathrm{v}}=21.3 \mathrm{~km} \mathrm{~s}^{-1}$.

4) The starburst has deposited significant amounts of energy into the disk, creating high velocity features and turbulence such that the line-of-sight velocity dispersion is much larger than the underlying rotational velocity due to the mass distribution of the galaxy. The starburst has had a more limited effect on the outer disk where rotational velocities dominate otherwise still significant turbulent motions.

5) In addition, NGC 1569 contains a significant amount of HI gas at discrepant, high velocities. Components that can be separated from the regularly rotating gas already provide $10 \%$ of the total HI mass of the galaxy. At least some of the highvelocity $\mathrm{HI}$ appears to be associated with the $\mathrm{H} \alpha$ filaments, but it is unclear whether it respresents the neutral component of the outflow. No evidence was found for a large bubble associated with the high-velocity HI. It is equally likely that at least part of the high-velocity HI clouds is associated with the HI companion and bridge linking this companion with NGC 1569. In that case, infall rather than outflow might be the cause of the discrepant velocities.

\section{References}

Castles, J., McKeith, C. D., \& Greve, A. 1991, Vistas Astron., 34, 187 Della Ceca, R., Griffiths, R. E., Heckman, T. H., \& Mackenty, J. W. 1996, ApJ, 469, 662

De Marchi, G., Clampin, M., Greggio, L., et al. 1997, ApJ, 479, L27

de Vaucouleurs, G., de Vaucouleurs, A., \& Pence, W. 1974, ApJ, 194, L119

de Vaucouleurs, G., de Vaucouleurs, A., Corwin, H. G., et al. 1991, Third Reference Catalogue (Springer Verlag, NY)

Drissen, L., \& Roy, J.-R. 1994, PASP, 106, 974

Greggio, L., Tosi, M., Clampin, M., et al. 1998, ApJ, 504, 725

Greve, A., Becker, R., Johansson, L. E. B., \& McKeith, C. D. 1996, A\&A, 312, 391

Heckman, T. M., Dahlem, M., Lehnert, M. D., Fabiano, G., \& Waller, W. H. 1995, ApJ, 448, 98

Högbom, J. A. 1974, A\&AS, 15, 417

Ho, L. C., \& Filippenko, A. V. 1996, ApJ, 466, L83

Huchtmeier, W. K., Karachentsev, I. D., \& Karachentsev, V. E. 2000, in Small Galaxy Groups, IAU Symp. 174, ed. M. J. Valtonen, \& C. Flynn, ASP Conf. Ser., 209, 158

Mac Low, M.-M., \& Ferrara, A. 1999, ApJ, 513, 142

Israel, F. P. 1980, A\&A, 90, 246

Israel, F. P. 1988, A\&A, 194, 24

Israel, F. P., \& De Bruyn, A. G. 1988, A\&A, 198, 109

Israel, F. P., \& Van Driel, W. 1990, A\&A, 236, 323

Kennicutt, R. C., \& Kent, S. M. 1983, AJ, 88, 1094

Leitherer, C., Schaerer, D., Goldader, J. D., et al. 1999, ApJS, 123, 3

Lisenfeld, U., Israel, F. P., Stil, J. M., \& Sievers, A. 2002, A\&A, 382, 860

Martin, C. L. 1998, ApJ, 506, 222

Martin, C. L. 1999, ApJ, 513, 156

Reakes, M. 1980, MNRAS, 192, 297

Stil, J. M., \& Israel, F. P. 1998, A\&A, 337, 64

Stil, J. M., \& Israel, F. P. 2002, A\&A, in press

Suchkov, A. A., Balsara, D. S., Heckman, T. M., \& Leitherer, C. 1994, ApJ, 430, 115

Tomita, A., Ohta, K., \& Saito, M. 1994, PASJ, 46, 335

Vallenari, A., \& Bomans, D. J. 1996, A\&A, 313, 713

Waller, W. H. 1991, ApJ, 370, 144 